\title{
Study Cellular Hypoxic Response under Cyclic Oxygen Gradients Generated in Microfluidic Devices Using Real-Time Fluorescence Imaging
}

Dao-Ming Chang

Academia Sinica

Yi-Chung Tung ( $\nabla$ tungy@gate.sinica.edu.tw )

Academia Sinica

\section{Research Article}

Keywords: cyclic oxygen gradients, cell culture system, computer-controlled gas supply system, microfluidic

Posted Date: April 5th, 2021

DOI: https://doi.org/10.21203/rs.3.rs-382697/v1

License: (c) (i) This work is licensed under a Creative Commons Attribution 4.0 International License.

Read Full License 


\title{
Study Cellular Hypoxic Response under Cyclic Oxygen Gradients Generated in Microfluidic Devices Using Real-Time Fluorescence Imaging
}

\author{
Dao-Ming Chang ${ }^{1}$ and Yi-Chung Tung ${ }^{1,2, *}$ \\ ${ }^{1}$ Academia Sinica, Research Center for Applied Science, Taipei, 11529, Taiwan \\ ${ }^{2}$ Chang Gung University, College of Engineering, Taoyuan, 33302, Taiwan \\ *tungy@gate.sinica.edu.tw
}

\begin{abstract}
In this paper, an integrated approach to study cellular hypoxic response under cyclic oxygen gradients is developed. In the experiments, a cell culture system based on a microfluidic device is constructed to generate cyclic oxygen gradients with fast response. The oxygen gradient is generated by alternatively introducing gases with specific compositions by a computer-controlled gas supply system into the microfluidic channels next to the cell culture one separated by thin channel walls. Observation of cellular hypoxic responses is performed using real-time fluorescence imaging of dyes sensitive to extra- and intra-cellular oxygen tensions as well as intracellular calcium concentrations. The capability of consistent cyclic oxygen gradient generation is confirmed by characterizing spatiotemporal oxygen tension profiles within the device. In the cell experiments, cellular hypoxic responses of human aortic smooth muscle cells (AoSMCs) and lung carcinoma epithelium (A549) cells including intracellular oxygen and calcium levels are measured. The relationships between the oxygen probe intensity and extracellular oxygen tension for both cell types are highly linear, and difference between the intensity measured from the two cell types is observed between 4 to $8 \% \mathrm{O}_{2}$ culture environment. In addition, both cell types show detectable intracellular calcium concentration variation when the environmental $\mathrm{O}_{2}$ varies between normoxia and lower than $4 \%$. The AoSMCs and A549 cells show decrease and increase of the intracellular calcium concentration during hypoxic response when facing change from normoxia to hypoxia environments, respectively. With the capability of real-time cellular responses monitoring under cyclic oxygen gradients, the developed approach provides a useful scheme to investigate cellular hypoxic response in vitro under microenvironments mimicking various in vivo physiological and pathological conditions.
\end{abstract}




\section{Introduction}

Molecular oxygen $\left(\mathrm{O}_{2}\right)$ is essential for cell survival. While the atmospheric oxygen concentration remains constant around $20.9 \%$, the concentration among different parts of human tissues can be as high as $19.7 \%$ in tracheus, or as low as about $1 \%$ in the superficial region of skin. ${ }^{1}$ When the oxygen availability is compromised, cells act accordingly to sustain life. The collective actions are called hypoxic responses. One can experience reduced oxygen availability either in a daily life in the form of exercise and altitude change, or pathologically in the form of heart failure, pulmonary disease, anemia or cancer. ${ }^{2-9}$ Studying hypoxic response and underlying oxygen sensing mechanism is therefore important. Even though different kinds of cells have distinct hypoxic responses, researchers have found that most hypoxic responses are orchestrated by hypoxia-inducible factors (HIFs), with the most studied subtype being HIF- $1 .{ }^{10}$ Other compelling oxygen sensing mechanisms involving reactive oxygen species (ROS) generation (redox-balancing) and ion channel activity are also reported. ${ }^{11}$

In both physiological and pathological conditions, hypoxic responses play key roles in regulating various biological activities. For instance, hypoxic pulmonary vasoconstriction (HPV) is one of the essential physiological hypoxic responses. Pulmonary arteries constrict in response to alveolar hypoxia to improve gas exchange in lungs and blood delivery to systemic tissues. ${ }^{12}$ The vasoconstriction is made possible by the hypoxia-induced increase of cytosolic calcium in pulmonary artery smooth muscle cells (PASMCs) in a biphasic process. A fast-acting first phase is followed by a slow but substantial second phase. The second phase of HPV is inhibited by the denudation of endothelial cell around PASMCs, but hypoxic-induced increase of cytosolic $\mathrm{Ca}^{2+}$ in PASMC is not compromised. The inhibition of second phase of HPV is regulated by the decrease of myofilament sensitivity to cytosolic $\mathrm{Ca}^{2+}$ in PASMC. ${ }^{13}$ Despite not conclusive, these observations suggest a central role of PASMC in HPV sensing mechanism. Similarly, the systemic arteries are regulated by oxygen tension. In contrast, systemic arteries dilate under hypoxia ${ }^{14}$, and the oxygen sensing mechanism of hypoxic-induced systemic arteries dilation is seldom studied. Previous studies find an increase of ROS contributed by mitochondria is involved in both hypoxia-induced pulmonary artery contraction and systemic artery dilation. ${ }^{12}$

In addition, tumor-induced hypoxia is observed among various types of cancers with average $1.5 \%$ to $2 \%$ of oxygen tensions in cancer biology. Tumor-induced hypoxia has been proven to enhance progression, metastasis, angiogenesis, and therapeutic resistance. ${ }^{15}$ As a result, targeting the hypoxic signalling mechanisms of cancer cells is believed to possess therapeutic values. Among studies regarding the mechanism of hypoxic signalling in cancer biology, HIFs are long considered to be of central roles. In chronic hypoxia in the core region of a solid tumor, drug targeting on HIF-1 $\alpha$ has been proven to be a promising therapeutic strategy. ${ }^{16,17}$ Moreover, under cyclic hypoxia condition in which the cell experiencing intermittent hypoxia and normoxia environments, recent studies show HIF-2 $\alpha$ is the dominant signalling factor for the fact that prolonged (>4-12hr) cyclic hypoxic/normoxic cycle enhances HIF-2 $\alpha$ but not HIF-1 $\alpha$ accumulation among different cancer cell lines. ${ }^{18}$ This observation suggests different signal transduction mechanisms may exist for chronic and intermittent hypoxia.

In order to study the hypoxic response and oxygen sensing mechanism of cells, precise and consistent control of extracellular oxygen tension is desired. In conventional broadly conducted cell culture experiments, cells are cultured using bicarbonate-buffered medium at $37^{\circ} \mathrm{C}$ with $5 \% \mathrm{CO}_{2}$ enriched ambient air, and humidity under non-condensing condition in incubators. When performing hypoxic experiments using a gas control incubator/chamber, typical response time taken to reach steady state is within several tens of minutes to an hour, depending on chamber size and setpoint value. After the culture environment reaches setpoint, it further takes additional several minutes to hours to equilibrate the dissolved oxygen into gas phase. ${ }^{19}$ Despite the gas-controlling incubation/chamber has been proven to be useful for chronic hypoxic research over last 2 decades, a cell culture system capable of manipulating oxygen tension at faster response is needed to explore acute hypoxic response and sensing mechanisms of the cells.

To overcome the challenges, an approach capable of reliably altering extracellular environments between different hypoxia levels and the normoxia condition with short response time is highly desired. Among various methods, microfluidics has been broadly exploited for cell culture with dynamic microenvironments due to its desired advantages including small sample volume and high surface area to volume ratio that can greatly help to reduce the response time required to reach equilibrium. For example, Polinkovsky et al. developed a microfluidic device capable of generating series of oxygen concentrations with short response time (in order of seconds) by on-chip gas mixing to study cellular responses. ${ }^{20}$ Also, Chen et al. exploited the spatially-confined chemical reaction method to rapidly establish oxygen gradients within the microfluidic channel within minutes for cell culture applications. ${ }^{21}$

In order to investigate temporal cellular hypoxic responses under various cyclic oxygen tensions, an integrated approach combining a microfluidic device, fluorescence dyes sensitive to extra/intra-cellular oxygen tensions and calcium concentration, and real-time fluorescence imaging is developed in this paper. In the experiments, a single-layered microfluidic device capable of generating cyclic oxygen gradients switching between hypoxic oxygen gradients and normoxic conditions within minutes is constructed for cell culture. The device is mainly made of PDMS due to its desired gas permeability, optical transparency, and cell compatibility. In the developed device, the oxygen gradient is generated by flowing gas with specific compositions into a microfluidic channel next to the cell culture one separated by thin walls. The gas flow is controlled using a computer-controlled 3-way solenoid valve. The single-layer device architecture with good optical transparency of PDMS and a glass substrate makes real-time observation on the acute hypoxic response of cells using microscopes feasible.

For the hypoxic response study, two types of cells: smooth muscle cells and epithelial cells are cultured inside the devices in the cell experiments. The function of the device is first characterized to confirm its capability for cyclic oxygen gradient 
generation. The intracellular oxygen tensions and calcium levels are then measured in a time-lapsed manner for the two distinct cell types under the generated cyclic oxygen gradients. The experimental results confirm the capability of the device to generate the cyclic oxygen gradients for the cell experiments. Furthermore, the cell experiments show the application of the real-time fluorescence imaging for study of cellular hypoxic responses under the cyclic oxygen gradients in variation of the intracellular oxygen tensions and calcium levels. The cell experimental results indicate distinct responses of the two tested cell types, and suggest the importance to investigate hypoxic responses of the cells in temporal domain. With the short response time and high throughput on control of oxygen tensions, and high compatibility to optical assays of the device, the developed approach integrating the real-time fluorescence imaging serves as an efficient method to explore cell kinetic responses under extracellular oxygen tension variation as well as critical oxygen tension triggering acute hypoxic responses and underlying mechanisms for various biomedical studies.

\section{Materials and Method}

\section{Device design and fabrication}

The microfluidic device capable of generating cyclic oxygen gradients is made of an elastomeric material, polydimethylsiloxane (PDMS), because of its high gas permeability and optical transparency. The device is composed of a top layer with microfluidic channel patterns and a PDMS-coated glass bottom layer. Three sets of microfluidic channels are designed in the top layer including: one $1 \mathrm{~mm}$-wide middle channel for cell culture and two sets of $100 \mu \mathrm{m}$-wide side channels (Figure 1a). The channels are all designed with single inlets and outlets. Air or nitrogen gas is injected into the side channel from the inlet for cyclic oxygen gradient generation. The middle and side channels are separated by $60 \mu \mathrm{m}$-wide oxygen permeable PDMS thin walls to prevent direct gas bubbling into the medium while altering oxygen tensions in the middle channel.

The top layer is fabricated using the well-developed soft lithography replica molding process. A master mold with positive relief features of the microfluidic channels is fabricated on a 4 inch-silicon wafer using negative tone photoresist (SU8 2050, MicroChem Co.) by conventional optical photolithography. To enhance the easiness of demolding the cured PDMS from the mold, the fabricated mold is silanized using $1 \mathrm{H}, 1 \mathrm{H}, 2 \mathrm{H}, 2 \mathrm{H}$-perfluorooctyltrichlorosilane (78560-45-9, Alfa Aesar) for $30 \mathrm{~min}$ in a desiccator at room temperature. PDMS pre-polymer with 1:10 (w/w) of curing agent and base is mixed, poured on the mold, degassed in desiccator, and then cured at $60^{\circ} \mathrm{C}$ for more than 4 hours. After curing, the interconnection inlets and outlets are created by a biopsy punch with a diameter of $1.5 \mathrm{~mm}$. For the bottom layer fabrication, a glass slide is spin-coated with the aforementioned PDMS pre-polymer and cured at $60^{\circ} \mathrm{C}$ for more than 4 hours. Both layers are treated with oxygen plasma (PX-250, Nordson MARCH Co.) at $95 \mathrm{~W}$ for $35 \mathrm{~s}$, sealed against each other, and heated at $60^{\circ} \mathrm{C}$ for more than 4 hours to promote the bonding between the layers. Figure $1 \mathrm{~b}$ shows the experimental photos of the fabricated devices filled with food dyes. The simple device design and fabrication process make the device easy to set up for practical biological applications.

\section{Cell culture and seeding}

To demonstrate the capability of the integrated approach, two types of cells, smooth muscle cell and epithelial cell, are cultured and investigated in the experiments. First, human aortic smooth muscle cell (AoSMC) (CC-2571, Lonza) is exploited. The cells are cultured with smooth muscle cell basal medium (CC-3182, Lonza) with growth factor supplements (CC-4149, Lonza) and maintained as following process. The attached cells are trypsinized by trypLE (12604013, Gibco), centrifuged under $209 \times \mathrm{g}$ for 5 minutes and resuspended into $1 \mathrm{~mL}$ of the prewarmed medium. After cell counting, $7 \times 10^{4}$ cells are seeded into a $25 \mathrm{~cm}^{2}$ flask (156367, Thermo Scientific). The medium is changed every 2-3 days, and the cells are passaged when reaching $70-80 \%$ confluency, which takes around 1 week. For optimized cell condition and consistent results, the AoSMCs used in the experiments are all under 10 passages. In addition, human alveolar basal epithelial cell (A549, ATCC) is also cultured in the experiments for comparison. The cells are cultured in DMEM (10566, Gibco) with 10\% v/v FBS (10082, Gibco) and 1\% v/v antibiotics (15240, Gibco). The cell stocks are all maintained in a $37^{\circ} \mathrm{C}$ humidified incubator supplied with $5 \% \mathrm{CO}_{2}$. Cell suspension used for the cell culture in the devices is prepared by a process similar to routine passage, except that after centrifugation, cell suspension is made concentrated by resuspending the pellets with $100 \mu \mathrm{L}$ of the prewarmed medium. After cell number counting, concentrated cell suspension is further diluted into final concentrations of 5,000 cells/ $\mu \mathrm{L}$ and 10,000 cells $/ \mu \mathrm{L}$ for AoSMC and A549, respectively.

Before seeding the cells into the microfluidic device, the cell culture channel is first made hydrophilic by oxygen plasma treatment at $95 \mathrm{~W}$ for $35 \mathrm{~s}$. The channel is then coated with cellular matrix protein, fibronectin (FC010, Millipore), with a concentration of $100 \mu \mathrm{g} / \mathrm{ml}$ to promote the cell attachment. The cell suspension is then pipetted into the cell culture channel from the inlet. Less than $20 \mu \mathrm{L}$ of cell suspension is needed for single device seeding. The cells are cultured inside the device for more than 18 hours under static condition to ensure the proper adhesion of the cells on the substrate for following experiments.

\section{Fluorescence imaging analysis}

For study of cellular responses under cyclic oxygen gradients, two types of analysis are performed in the experiments. First, 
the intracellular oxygen tension is characterized using a commercially available MitoXpress Intra intracellular oxygen assay (MX-300, Agilent Technologies, Inc.). The reagent is diluted with the complete cell culture medium at volumetric ratio of 1:10 and applied into the cell-seeded device while the cells are attached onto the culture surface. The cells are further incubated in the diluted reagent overnight.

In addition, intracellular calcium concentration is also investigated in the experiments. Calcium ion $\left(\mathrm{Ca}^{2+}\right)$ is an essential intracellular messenger regulating various cell functions. Monitoring calcium level under temporal and spatial oxygen tension change potentially give insights of understanding the fast-acting oxygen sensing mechanism and hypoxic response behind. In the $\mathrm{Ca}^{2+}$ measurement experiments, after the cell seeding and overnight culture, the growth medium inside the device is replaced by the complete medium with $3 \mu \mathrm{M}$ of X-Rhod-1 Acetoxymethyl (AM) ester (X14210, Invitrogen). The cells are incubated for $30 \mathrm{~min}$ at $37^{\circ} \mathrm{C}$ for dye loading. After the dye loading, the cells are then washed using the complete medium and incubated at $37^{\circ} \mathrm{C}$ for additional $30 \mathrm{~min}$ to make AM ester completely hydrolyzed and thus make X-Rhod-1 fluoresces.

The stained cells cultured in the microfluidic devices are imaged using an inverted fluorescence microscope (DM IL, Leica Microsystems, Wetzlar, Germany) equipped with a CCD camera (DFX360, Leica Microsystems), a multi-color LED light source (X-Cite XLED1, Excelitas Technologies Corp., Waltham, MA), and a 10X objective (HI Plan, Leica Microsystems). Control of the microscope and automatic time-lapse image acquisition are achieved by a commercially available microscopy automation and image analysis software (Metamorph Version 7.7, Molecular Devices, LLC, San Jose, CA). For the intracellular oxygen characterization, a multiband filter cube suitable for the MitoXpress Intra imaging (380-395 nm band-pass excitation filter, $575 \mathrm{~nm}$-dichromatic mirror, and 580-650 nm band-pass emission filter) is exploited (Semrock DA-FI-TR-3X-A-000, IDEX Health \& Science, Rochester, NY) (Figure 1c). The imaging parameters are set as UV LED excitation at $\%$ intensity, $200 \mathrm{~ms}$-exposure, 4 times frame averaging, and $2 \times 2$ binning. To minimize the photocytotoxicity during the imaging, the images are captured every 1 minute for an hour. For the intracellular calcium observation, the same multiband filter cube with a blue band-pass excitation filter (470-490 nm), $500 \mathrm{~nm}$-dichromatic mirror, and 500-535 nm band-pass emission is exploited. The imaging parameters are set as blue LED excitation at 5\% intensity, $200 \mathrm{~ms}$-exposure, and $2 \times 2$ binning. For AoSMC and A549, the images are captured every 2 seconds and 30 seconds for an hour, respectively.

The captured time-lapsed fluorescence images are quantitatively analyzed using an imaging analysis software ImageJ (Ver. 1.52p, NIH) with Time Series Analyzer plugin (https://imagej.nih.gov/ij/plugins/time-series.html) and further visualized by a mathematics software, MATLAB (R2017a, MathWorks). All time-lapsed fluorescence intensity values are reported by fold change comparing to the intensity obtained from the first frame in the same experiment (normalized fluorescence intensity, $\mathrm{Fi} / \mathrm{F} 0$ ). For intracellular oxygen measurement, baseline correction is applied by fitting the time-lapsed normalized fluorescence intensity data against asymmetric truncated quadratic function to compensate the effect of photobleaching.

\section{Oxygen gradient generation and characterization}

In order to generate cyclic oxygen gradients within the middle cell culture channel, mixed air (air with $5 \% \mathrm{CO}_{2}$ ) is constantly introduced into one side channel and pure nitrogen (i.e., $0 \% \mathrm{O}_{2}$ ) and the mixed air are alternatively introduced into another side channel with a period of 10 minutes. Both mixed air and nitrogen gases are injected into the side channels with inlet pressures larger than 0.4 bar. The alternation between the gases is accomplished by switching the power of a 3-way solenoid valve (ZHV-0519, ZonHen Co., Ltd.) on/off using a personal computer-controlled DC power supply (E3631A, Agilent Technologies, Inc., Santa Clara, CA) via a GPIB interface as shown in Figure 1c. In the experiments, the solenoid valve is operated under $5 \mathrm{~V} / 0.2 \mathrm{~A}$.

In order to characterize the oxygen tensions generated in the middle culture channel, an oxygen sensitive fluorescence dye, Tris(2,2'-bipyridyl)dichlororuthenium(II) hexahydrate (RTDP) (224758, Sigma) is perfused into the middle channel with a flow rate of $10 \mu \mathrm{l} / \mathrm{min}$. For the RTDP intensity profile measurement, 25\% power blue LED illumination, $200 \mathrm{~ms}$-exposure, no average and binning are set as imaging parameters. The fluorescence intensity of the dye is quenched by the presence of oxygen; therefore, the spatial distribution of the oxygen tension inside the channel can be estimated by measuring the intensity profile. ${ }^{22}$ The relationship between the fluorescence intensity and oxygen tension is given by Stern-Volmer equation as $\mathrm{I}_{\mathrm{f}}^{0} / \mathrm{I}_{\mathrm{f}}=1+\mathrm{K} \cdot[\mathrm{Q}]$, where $\mathrm{I}_{\mathrm{f}}$ and $\mathrm{I}_{\mathrm{f}}^{0}$ are the fluorescence intensity values with and without quencher (oxygen), respectively. $\mathrm{K}$ represents the quenching coefficient, and $[\mathrm{Q}]$ is the concentration of the quencher (oxygen). The quenching coefficient $\mathrm{K}$ is calibrated by introducing pure nitrogen and oxygen representing either $0 \%$ and $100 \%$ oxygen tension into the side channels, respectively. ${ }^{21}$ The calibrated quenching coefficient is further validated by calculating the oxygen tension under mixed air gas (ambient air with $5 \% \mathrm{CO}_{2}$ ).

\section{Results and discussion}

\section{Characterization of cyclic oxygen gradient generation}

Oxygen gradient is generated and eliminated by alternatively introducing the mixed air (air with $\left.5 \% \mathrm{CO}_{2}\right)\left(\mathrm{N}_{2} \mathrm{OFF}\right.$ ) and pure nitrogen $\left(\mathrm{N}_{2} \mathrm{ON}\right)$ into the left side channel while constantly flowing the mixed air into the right side channel in the experiments. The control of alternation between the gases is achieved using the computer-controlled three-way solenoid valve (Figure 1).

The oxygen tension profiles characterized using the oxygen sensitive dye in the cell culture channel are shown in Figure 
2. Figure 2(a) and 2(b) show the oxygen tension distribution within the cell culture channel when introducing the mixed air and the pure nitrogen into the life side channel, respectively. An animation of the real-time oxygen tension profile change in the cell culture channel is available in the Supporting Information. When flowing the mixed air into both side channels, the oxygen tension profile is more uniform across the width of the cell culture channel with an average value of $17.2 \%$ as shown in Figure 2(a). Majority of the oxygen tension within the channel (>90\% of the area) lies between 17 and 20\%, and the lowest value occurs at the center position of the channel due to long diffusion length. In contrast, after switching from the mixed air to nitrogen gas into the left side channel, the oxygen tension within the cell culture channel monotonically decreases from right to left sides ranging from $0.3 \%$ to $18.3 \%$ as shown in Figure 2 (b). The plot shows that the oxygen gradient can be successfully established using the developed device and method. During the cyclic oxygen gradient generation process, the two oxygen tension profiles are switched every 10 minutes.

In order to quantify the generated cyclic oxygen gradients and their temporal patterns, the oxygen tension profiles along the channel width at different time points under the $\mathrm{N}_{2}$ OFF and ON conditions are plotted as Figure 2(c). The plot shows that the oxygen tension profiles are consistent under both conditions, and the differences between the profiles under the $\mathrm{N}_{2}$ OFF and $\mathrm{N}_{2} \mathrm{ON}$ conditions are less than $0.83 \%$ and $0.64 \%$, respectively. To further observe the dynamic of the oxygen tension variation in the temporal domain, the cell culture channel is divided into 8 regions for quantitative analysis. The temporal variation of the average oxygen tension within each zone is plotted in Figure 2(d). The plot shows that the average oxygen tension cycles between $19.5 \%$ and $1.6 \%$ within the region closest to the left side channel (R1). In contrast, for the most distal region from the left side channel (R8), the oxygen tension cycles between $18.1 \%$ and $15.5 \%$. The average oxygen tension values vary from different hypoxia degrees ranging from $1.6 \%$ to $19.5 \%$ from left (R1) to right (R8) side of the channel to normoxia (higher than 18.9\%) in a period of 20 minutes. In addition, the temporal variation patterns in all regions within each period are similar to those in other periods. The results indicate that consistent cyclic oxygen gradients can be successfully generated using the device and further exploited for cell culture applications.

\section{Intracellular oxygen tension characterization}

For real-time observation of intracellular oxygen tensions, AoSMC and A549 cell are stained with fluorescent intracellular oxygen probe (MitoXPress Intra). The fluorescence of the probe is quenched by molecular oxygen; therefore, decrease in fluorescence intensity indicates increase of intracellular oxygen tension. Figure 3 and 4 show the phase contrast and fluorescence images, and quantitative fluorescence intensity profiles along the width of the channel in the AoSMC and A549 cell culture experiments, respectively. The phase contrast and fluorescent images are taken before (Fig. 3a and 4a) and after (Fig. $3 \mathrm{~b}$ and $4 \mathrm{~b}$ ) the 1-hour time-lapsed imaging process with 1 minute-intervals. Comparing the images before and after the 1-hour imaging, it is observed that both cells remain attached on the substrates. However, slightly round up feature of the cells are observed after the measurements. The feature is susceptible to be the phototoxicity effect caused by the probe/molecular oxygen quenching process which is known to produce reactive oxygen species (ROS, e.g., singlet oxygen). ${ }^{23}$

Furthermore, the fluorescent images under both $\mathrm{N}_{2}$ OFF (normoxia) and $\mathrm{N}_{2} \mathrm{ON}$ (oxygen gradient) conditions are quantitatively analyzed, and the fluorescence intensity profiles are plotted in Figure $3 \mathrm{c}$ and $4 \mathrm{c}$ for the AoSMC and A549 cells, respectively. The plots show that the fluorescence intensity of the oxygen sensitive dye is higher for the cells closer to the left side channel under the oxygen gradient condition when comparing to the normoxic control ones. This suggesting the lower intracellular oxygen tension of the cells on the left side when the $\mathrm{N}_{2}$ is on. In contrast, the fluorescence intensity for the cells close to the right side-channel remain similar for both conditions indicating similar intracellular oxygen tension. The results confirm that the intracellular oxygen tensions vary from low to high from left to right following the trends of the oxygen gradient generated in the cell culture channel.

In order to quantify the intracellular oxygen tension variation during the alternative normoxia $\left(\mathrm{N}_{2} \mathrm{OFF}\right)$ and oxygen gradient $\left(\mathrm{N}_{2} \mathrm{ON}\right)$ conditions, the average intensities of the oxygen sensitive dye within the cells located in eight different regions across the channel width (left to right: R1 to R8) during the experimental period are plotted as shown in Figure 5(a) and (b) for AoSMC and A549 cells, respectively. The figures show that the normalized fluorescence intensity $\left(\mathrm{F}_{\mathrm{i}} / \mathrm{F}_{0}\right)$ of the intracellular oxygen tension probe increases when the gas introduced into the left side channel changed from the mixed air to $\mathrm{N}_{2}$, i.e., resulting in change from the normoxia to oxygen gradient conditions in the cell culture channel. The intensity increase indicates that the intracellular oxygen tension decreases due to the surrounding extracellular environmental oxygen tension decrease. In addition, the fluorescence intensities within the cells located in the regions closer to the left side-channel with smaller region numbers are higher comparing to those within the cells in the regions further away from the left side-channel under the oxygen gradient condition. The results indicate the cells have lower intracellular oxygen tensions when exposed to microenvironments with lower oxygen tension suggesting that the intracellular oxygen tensions of both AoSMC and A549 cells can be modulated by the environmental oxygen microenvironments.

In order to compare the temporal hypoxic response of AoSMC and A549 cells, the normalized fluorescence intensities within the cells located in different regions within a period of condition alternation are plotted in Fig. 5(c). The plots show that both cells located in regions 1 and region 4-8 have similar intracellular oxygen tension responses with similar normalized fluorescence intensity variation patterns. Interestingly, the fluorescence intensities of the oxygen sensitive probes within the cells located in region 2 and 3 are slightly different between two cell types. In region 2, the normalized intensity within the 
A549 cells rises slightly faster and reach higher endpoint value than that within the AoSMC when introducing the nitrogen into the side channel for oxygen gradient generation. In contrast, in region 3, the intensity within the A549 cells rises slightly slower and reach lower endpoint value than that within the AoSMC during the nitrogen introduction. The results suggest the A549 reaches higher and lower intracellular hypoxia level in the region 2 and 3 comparing to the AoSMC, respectively. Also, the results indicate a critical hypoxia level that the two type cells respond to the extracellular oxygen microenvironments differently.

For identification of the critical oxygen tension value, the normalized fluorescence intensity within the AoSMC and A549 cells under different extracellular oxygen tensions during generation of cyclic oxygen gradients are plotted as shown in Fig. 5(d). The plot shows that most of the intensities within the two cell types overlapped for most of the oxygen tension values, and the relationships between the intensity and the oxygen tension are highly linear $\left(\mathrm{R}^{2}=0.931\right.$ and 0.918 for linear fitting of data from the AoSMC and A549 cells, respectively) for both cell types. The linear relation suggests that both cells vary their intracellular oxygen tension according to the extracellular oxygen microenvironments. Interestingly, the two cell types show different fluorescence intensity when the extracellular oxygen tension is between approximately $4 \%$ and $8 \%$, which are the oxygen tension within the aforementioned regions 2 and 3 during the oxygen gradient generation periods. In addition, the oxygen tension of approximately $6 \%$ is the value making the two cell types respond differently to the extracellular oxygen microenvironments. The future detail study is required to better investigate the underlying molecular mechanisms resulting in the different intracellular oxygen responses between the two cell types. The study may further help biologists understand the temporal hypoxic responses of the cells to the extracellular oxygen stimulations.

Furthermore, the kinetic hypoxic response of the intracellular oxygen tension to the extracellular one is investigated. The variation of the fluorescence intensities within the cells located in regions 1 to 4 during a period of deoxygenation $\left(\mathrm{N}_{2} \mathrm{OFF}\right.$ to $\mathrm{ON}$ ) and reoxygenation $\left(\mathrm{N}_{2} \mathrm{OFF}\right.$ to $\mathrm{ON}$ ) processes are plotted in Fig. 5(e). The plots show that the larger difference between the fluorescence intensity deviation between deoxygenation and reoxygenation when the extracellular oxygen tension cycles between higher hypoxia level and normoxia. For example, the deviation in fluorescence intensities of the intracellular oxygen tension-sensitive dye within the AoSMC and A549 cells located in region 1 is larger than those located in region 2, 3, and 4. The results suggest that the cells take longer time to response the extracellular oxygen tension variation with a larger range. The transient and kinetic hypoxic response of the cells to the extracellular oxygen variation has seldom systematically studied due to the technical limitations. The demonstrated results shown in this paper confirm the functionality of the developed device, and it can be further applied to investigate underlying mechanisms when the cells facing various oxygen microenvironments.

\section{Intracellular calcium level characterization}

For the observation of intracellular calcium level under cyclic oxygen gradients, both AoSMC and A549 cells are cultured under cycles of 10 min normoxia $\left(\mathrm{N}_{2} \mathrm{OFF}\right) / 10$ min oxygen gradient $\left(\mathrm{N}_{2} \mathrm{ON}\right)$ for 60 minutes. During the oxygen tension change, the calcium level is monitored via imaging of fluorescent active intracellular calcium indicator X-Rhod-1. Figure 6(a) and (b) show the phase contrast and fluorescence images of the AoSMCs and A549 cells stained with the indicator after the experiments, respectively. The images show that no apparent cell morphology change is observed for both cell types after the measurements.

Figure 7 shows the quantitative analysis results of the intracellular calcium concentration variation of the AoSMC and A549 cells located in different regions under the normoxia and cyclic oxygen gradient stimulation. For AoSMC, the cells cultured under both conditions show the increasing cytosolic calcium concentration during the 60 minute-culture inside the microfluidic devices. In addition, the cells located at R1 and R2 exhibits detectable intracellular calcium fluctuation under cyclic oxygen gradient stimulation. When the cellular environment is reoxygenated, i.e, when introducing the mixed air into the left side channel $\left(\mathrm{N}_{2} \mathrm{ON}\right.$ to $\left.\mathrm{OFF}\right)$, increase of the intracellular cytosolic calcium concentration is observed. In contrast, the intracellular cytosolic calcium concentration decreases when the cellular environment is deoxygenated, i.e., when the pure nitrogen is introduced into the side channel ( $\mathrm{N}_{2} \mathrm{OFF}$ to $\left.\mathrm{ON}\right)$.

In comparison, the A549 cells cultured under both conditions show the slightly decreasing cytosolic calcium concentration during the 60 minute-culture inside the microfluidic devices. Although the A549 cells located in the R1 and R2 also shows detectable intracellular calcium concentration fluctuation, the amplitude of the variation is smaller than those observed on the AoSMCs. Interestingly, when the cellular environment is reoxygenated and deoxygenated, decrease and increase of the intracellular cytosolic calcium concentration is observed, respectively. The trends are opposite to those observed on the AoSMCs, and the discrepancy has never been discussed in literature before and requires further study.

Even though it is not conclusive whether this calcium fluctuation observed on the AoSMCs contributes to muscle movements directly or not, it shed some light that possible oxygen tension dependent or feedback regulatory mechanisms exist on the AoSMC, which had been considered solely being an actuator orchestrated by endothelial cells and paracrine signaling. ${ }^{24}$ For the lung epithelial (A549) cells, oxygen dependent intracellular calcium fluctuation is observed as well even though the physiological meaning of the phenomena remains unclear. From the observation from both cell types, it shows that the hypoxia level with extracellular oxygen tension as low as $6 \%$ (according to the calibration value for R2) can trigger the intracellular calcium fluctuation for both cell types.

\section{Conclusions}

In this paper, an integrated approach combining microfluidic devices and real-time fluorescence imaging is developed 
to study cellular hypoxic response under cyclic oxygen gradients. To demonstrate the capability of the approach, we measure the intracellular oxygen tension and calcium level of two distinct human cells (AoSMC and A549 cell) and discuss their differences. The intracellular oxygen tension measurement indicates that, even though extracellular and intracellular oxygen tension is closely related, different types of cell can still exhibit different intracellular oxygen tension value in certain extracellular oxygen tension ( $4 \%$ to $8 \%$ ) that is not necessary to be the normoxic and hypoxic value ( 20.9 and $1 \%$, respectively) conventionally used. The intracellular calcium tension measurement on AoSMC shows that the reoxygenation from hypoxic $(<6 \%)$ to normoxic $(21 \%)$ environment triggers increase of intracellular calcium on AoSMC cells, which implies AoSMC, instead of being solely a vessel contraction/dilation actuator, has its own standalone roles on blood pressure regulation. With the two demonstrated applications on monitoring intracellular oxygen tension and the calcium level variation, it is confirmed that the real-time imaging can be exploited to study kinetic cellular hypoxic response due to the simple design and great optical transparency of the device. With the demonstrated functionalities and capabilities, the developed method can pave a way to study seldom explored kinetic cellular hypoxic response that is essential in various physiological and pathological conditions.

\section{Acknowledgements}

This paper is based on the work supported by the Taiwan Ministry of Science and Technology (MOST 107-2628-E-001-003MY3, 108-2221-E-001-003 and 109-2221-E-001-002-MY2), and the Academia Sinica Career Development Award (AS-CDA106-M07).

\section{Author Contribution Statements}

D.-M. C. and Y.-C. T. designed the study, conducted the experiments, analyzed the results, and wrote the manuscript. Both authors reviewed the manuscript.

\section{Additional information}

There are no conflicts to declare.

\section{References}

1 Carreau, A., El Hafny-Rahbi, B., Matejuk, A., Grillon, C. \& Kieda, C. Why is the partial oxygen pressure of human tissues a crucial parameter? Small molecules and hypoxia. J Cell Mol Med 15, 1239-1253, doi:10.1111/j.15824934.2011.01258.x (2011).

2 Jamshidi, N. \& Palsson, B. O. Systems biology of the human red blood cell. Blood Cells Mol Dis 36, 239-247, doi:10.1016/j.bcmd.2006.01.006 (2006).

3 Favier, F. B., Britto, F. A., Freyssenet, D. G., Bigard, X. A. \& Benoit, H. HIF-1-driven skeletal muscle adaptations to chronic hypoxia: molecular insights into muscle physiology. Cell Mol Life Sci 72, 4681-4696, doi:10.1007/s00018015-2025-9 (2015).

Conkin, J. \& Wessel, J. H., 3rd. Critique of the equivalent air altitude model. Aviat Space Environ Med 79, 975-982, doi:10.3357/asem.2331.2008 (2008).

5 Coppel, J., Hennis, P., Gilbert-Kawai, E. \& Grocott, M. P. The physiological effects of hypobaric hypoxia versus normobaric hypoxia: a systematic review of crossover trials. Extrem Physiol Med 4, 2, doi:10.1186/s13728-014-00216 (2015).

6 Ostadal, P. et al. Novel porcine model of acute severe cardiogenic shock developed by upper-body hypoxia. Physiol Res 65, 711-715, doi:10.33549/physiolres.933294 (2016).

7 Morita, Y., Chin-Yee, I., Yu, P., Sibbald, W. J. \& Martin, C. M. Critical oxygen delivery in conscious septic rats under stagnant or anemic hypoxia. Am J Respir Crit Care Med 167, 868-872, doi:10.1164/rccm.200205-490OC (2003).

8 Levy, P. et al. Obstructive sleep apnoea syndrome. Nat Rev Dis Primers 1, 15015, doi:10.1038/nrdp.2015.15 (2015).

9 Zhuan, B., Yu, Y., Yang, Z., Zhao, X. \& Li, P. Mechanisms of oxidative stress effects of the NADPH oxidase-ROSNF-kappaB transduction pathway and VPO1 on patients with chronic obstructive pulmonary disease combined with pulmonary hypertension. Eur Rev Med Pharmacol Sci 21, 3459-3464 (2017).

10 Ke, Q. \& Costa, M. Hypoxia-inducible factor-1 (HIF-1). Mol Pharmacol 70, 1469-1480, doi:10.1124/mol.106.027029 (2006).

11 Weir, E. K., Lopez-Barneo, J., Buckler, K. J. \& Archer, S. L. Acute oxygen-sensing mechanisms. N Engl J Med 353, 2042-2055, doi:10.1056/NEJMra050002 (2005).

12 Waypa, G. B. \& Schumacker, P. T. Hypoxia-induced changes in pulmonary and systemic vascular resistance: where is the O2 sensor? Respir Physiol Neurobiol 174, 201-211, doi:10.1016/j.resp.2010.08.007 (2010).

13 Robertson, T. P., Aaronson, P. I. \& Ward, J. P. Hypoxic vasoconstriction and intracellular Ca2+ in pulmonary arteries: 
evidence for PKC-independent $\mathrm{Ca} 2+$ sensitization. Am $J$ Physiol 268, H301-307, doi:10.1152/ajpheart.1995.268.1.H301 (1995).

14 Johnson, P. C. Autoregulation of blood flow. Circ Res 59, 483-495, doi:10.1161/01.res.59.5.483 (1986).

15 Muz, B., de la Puente, P., Azab, F. \& Azab, A. K. The role of hypoxia in cancer progression, angiogenesis, metastasis, and resistance to therapy. Hypoxia (Auckl) 3, 83-92, doi:10.2147/HP.S93413 (2015).

16 Burroughs, S. K. et al. Hypoxia inducible factor pathway inhibitors as anticancer therapeutics. Future Med Chem 5, 553-572, doi:10.4155/fmc.13.17 (2013).

17 Fallah, J. \& Rini, B. I. HIF Inhibitors: Status of Current Clinical Development. Curr Oncol Rep 21, 6, doi:10.1007/s11912-019-0752-z (2019).

18 Saxena, K. \& Jolly, M. K. Acute vs. Chronic vs. Cyclic Hypoxia: Their Differential Dynamics, Molecular Mechanisms, and Effects on Tumor Progression. Biomolecules 9, doi:10.3390/biom9080339 (2019).

19 Place, T. L., Domann, F. E. \& Case, A. J. Limitations of oxygen delivery to cells in culture: An underappreciated problem in basic and translational research. Free Radic Biol Med 113, 311-322, doi:10.1016/j.freeradbiomed.2017.10.003 (2017).

20 Polinkovsky, M., Gutierrez, E., Levchenko, A. \& Groisman, A. Fine temporal control of the medium gas content and acidity and on-chip generation of series of oxygen concentrations for cell cultures. Lab Chip 9, 1073-1084, doi:10.1039/b816191g (2009).

21 Chen, Y. A. et al. Generation of oxygen gradients in microfluidic devices for cell culture using spatially confined chemical reactions. Lab Chip 11, 3626-3633, doi:10.1039/c1lc20325h (2011).

22 Wolfbeis, O. S. Luminescent sensing and imaging of oxygen: fierce competition to the Clark electrode. Bioessays 37, 921-928, doi:10.1002/bies.201500002 (2015).

23 Dobrucki, J. W. Interaction of oxygen-sensitive luminescent probes $\mathrm{Ru}(\mathrm{phen})(3)(2+)$ and $\mathrm{Ru}(\mathrm{bipy})(3)(2+)$ with animal and plant cells in vitro. Mechanism of phototoxicity and conditions for non-invasive oxygen measurements. $J$ Photochem Photobiol B 65, 136-144, doi:10.1016/s1011-1344(01)00257-3 (2001).

24 Chan, C. K. \& Vanhoutte, P. M. Hypoxia, vascular smooth muscles and endothelium. Acta Pharm Sin B 3, 1-7, doi:10.1016/j.apsb.2012.12.007 (2013). 
Figures:

(a) Device Design

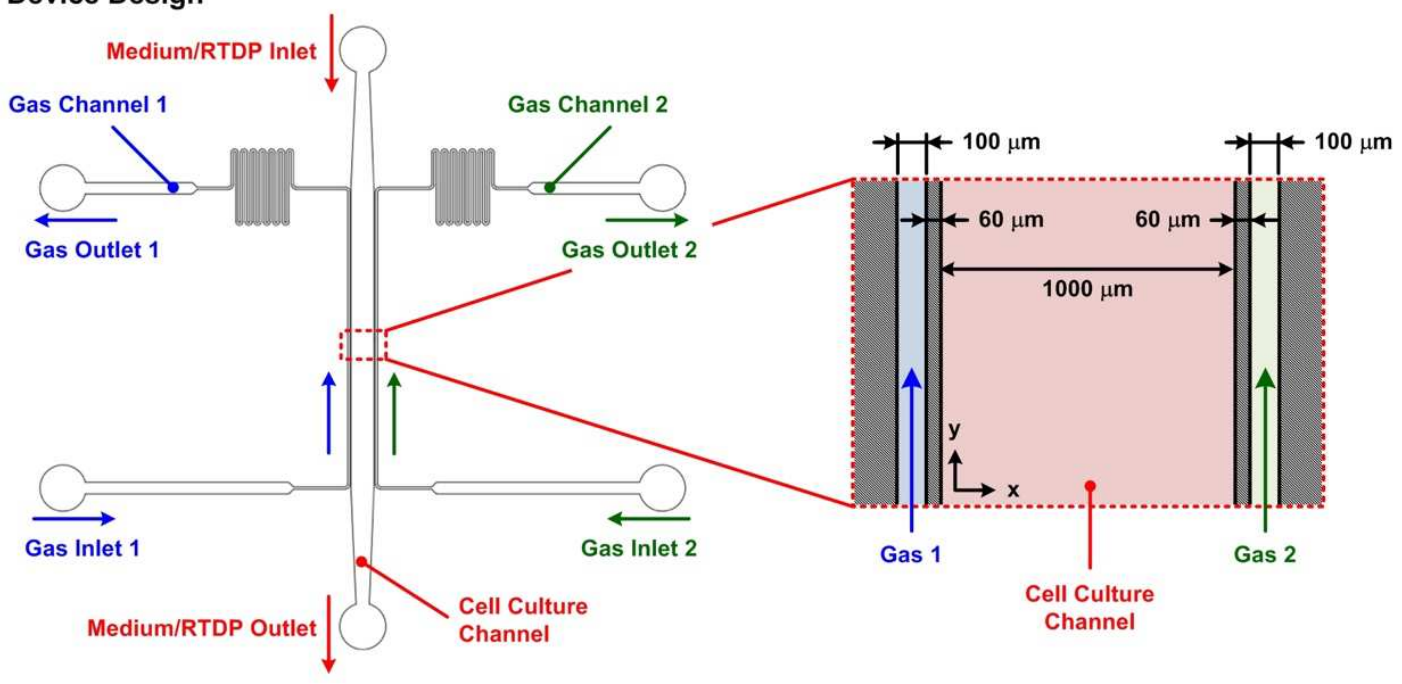

(b) Fabricated Device

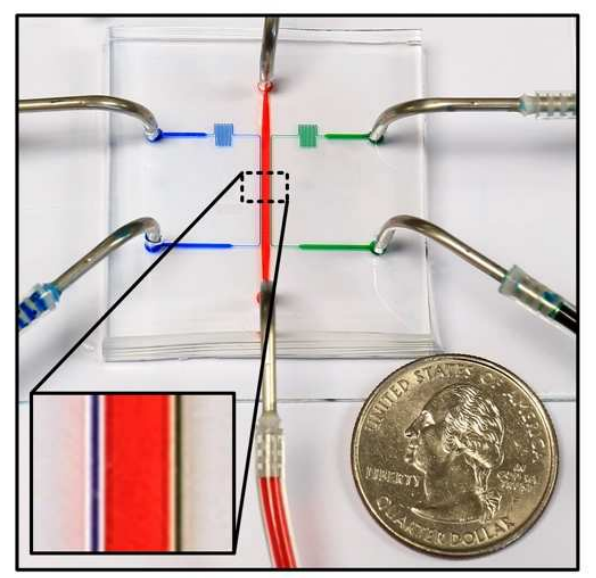

(c) Experimental Setup

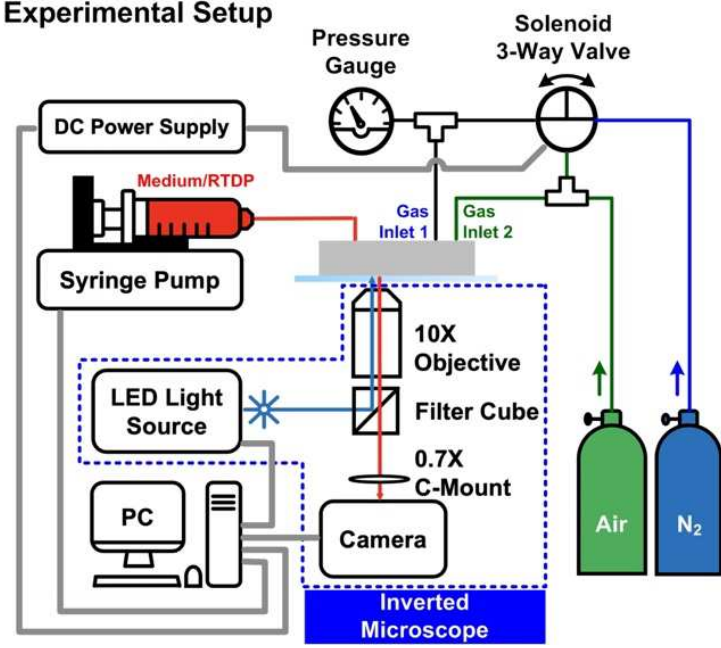

Figure 1. (a) Schematic of the PDMS microfluidic device capable of generating cyclic oxygen gradients. Three sets of microfluidic channels are designed in the device, including the middle cell culture channel and two side channels for oxygen gradient generation. The channels are separated by $60 \mu \mathrm{m}$-wide walls. Oxygen gradient in the cell culture channel is generated by purging pure nitrogen gas and air into the left (Inlet 1) and right (Inlet 2) side channels, respectively. (b) Experimental photos of the fabricated device filled with food dyes. (c) Experiment setup for generation of the cyclic oxygen gradients using the microfluidic device for cell culture experiments and observation. 
(a)

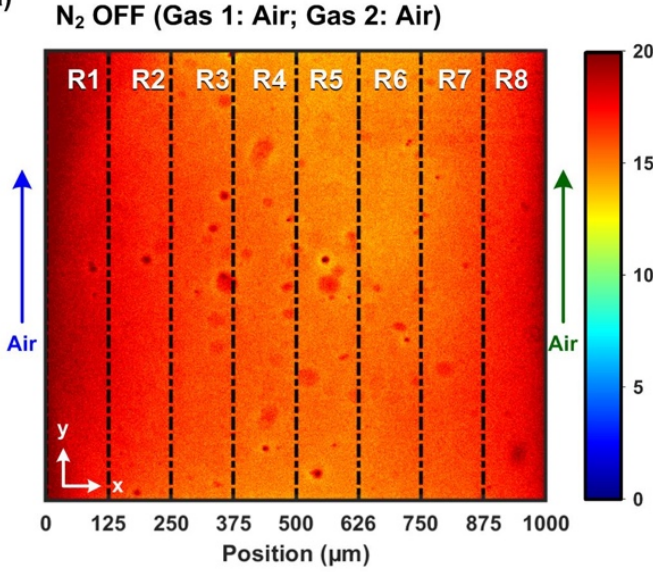

(c)

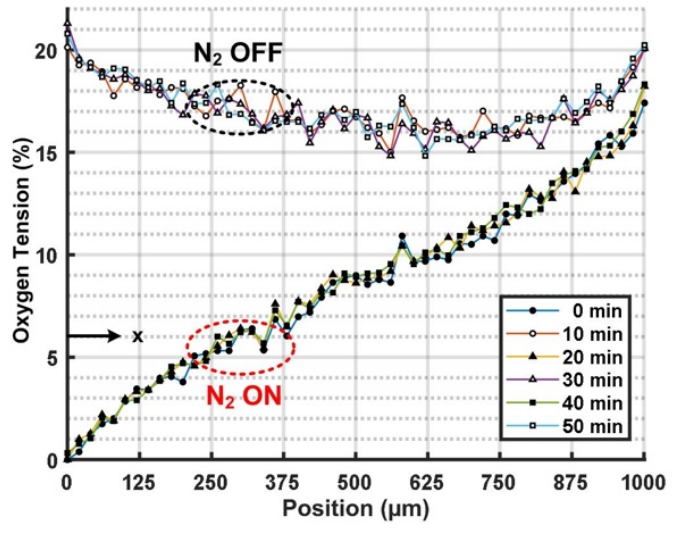

(b) $\mathrm{N}_{2}$ ON (Gas 1: $\mathrm{N}_{2}$; Gas 2: Air)

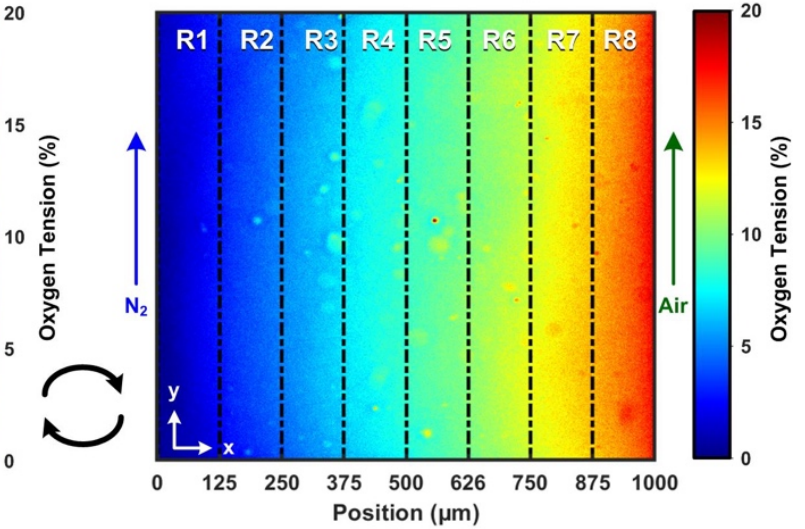

(d)

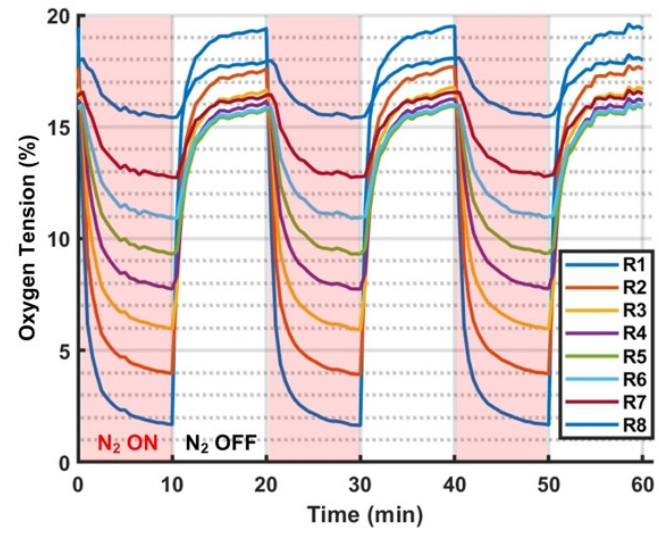

Figure 2. (a) and (b) are the characterized oxygen profiles without $\left(\mathrm{N}_{2} \mathrm{OFF}\right)$ and with $\left(\mathrm{N}_{2} \mathrm{ON}\right)$ oxygen gradients, respectively. (c) Line profiles show the consistent oxygen gradients (solid symbol lines) can be established when nitrogen gas is on $\left(\mathrm{N}_{2} \mathrm{ON}\right.$ ), and relative uniform normoxia conditions (hollow symbol lines) can be recovered by air purging $\left(\mathrm{N}_{2} \mathrm{OFF}\right.$ ). (d) The temporal variation of average oxygen tension values from the leftmost (R1) to the rightmost region (R8). Cyclic oxygen tension profiles in different regions show that the oxygen tensions are oscillated in the period of 20 minutes ( $10 \mathrm{~min} \mathrm{on} / 10 \mathrm{~min} \mathrm{off})$. 
(a)

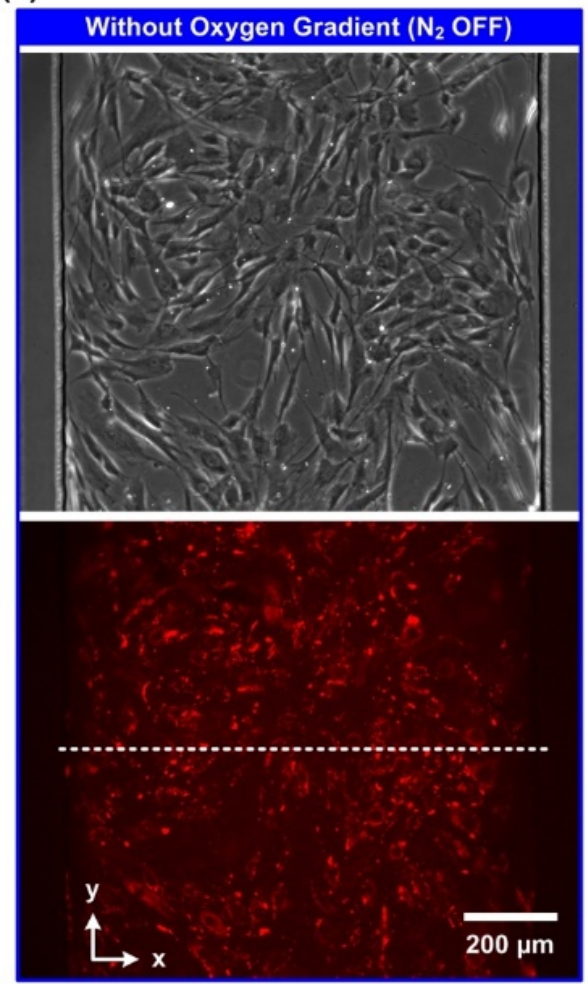

(b)

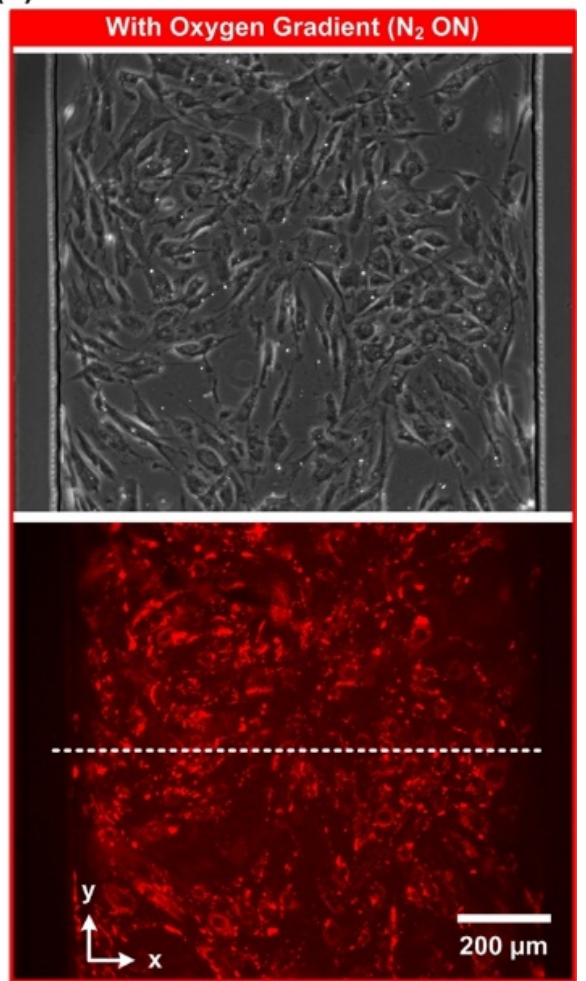

(c)

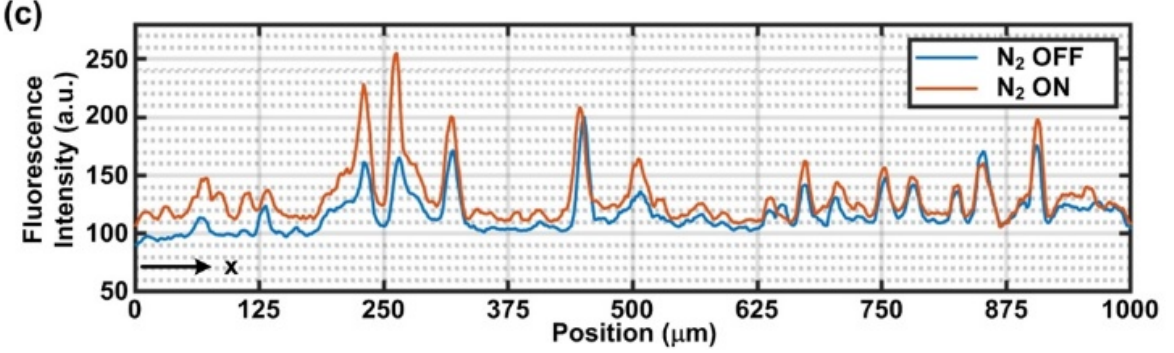

Figure 3. Bright field and fluorescence images of AoSMC treated with the intracellular oxygen sensitive reagent (MitoXpress Intra) under (a) normoxia $\left(\mathrm{N}_{2} \mathrm{OFF}\right)$ and (b) oxygen gradient $\left(\mathrm{N}_{2} \mathrm{ON}\right)$ conditions. (c) Representative line profiles of the fluorescence intensity across the width of the channel under the normoxia and oxygen gradient conditions. Comparing to the normoxia condition $\left(\mathrm{N}_{2} \mathrm{OFF}\right)$, higher fluorescence intensity on the left-hand side under the oxygen gradient condition $\left(\mathrm{N}_{2} \mathrm{ON}\right)$ indicates lower intracellular oxygen tension within the AoSMCs. 
(a)

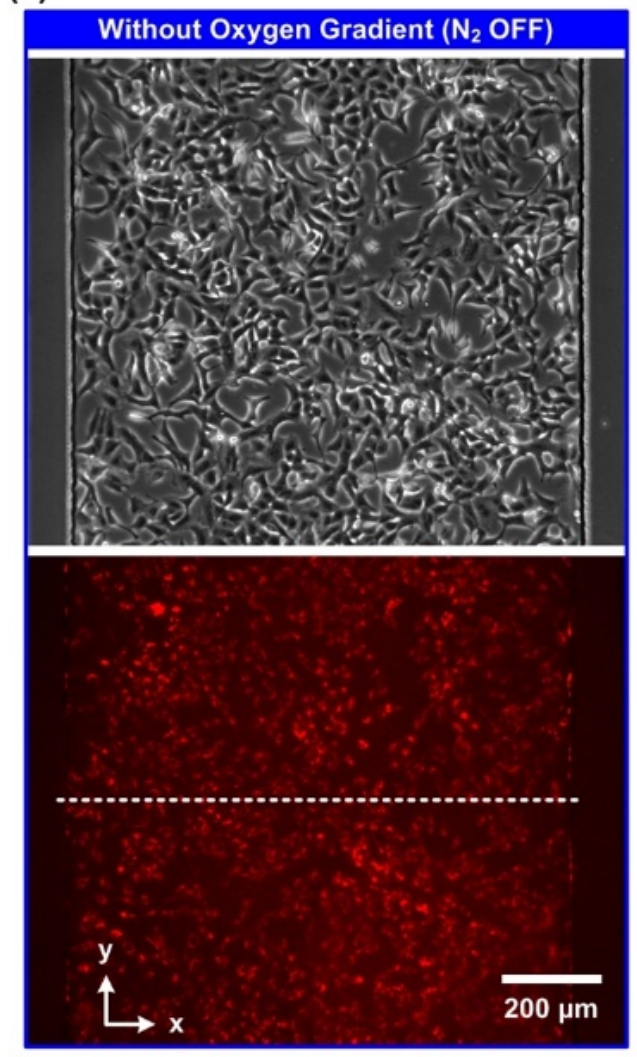

(b)

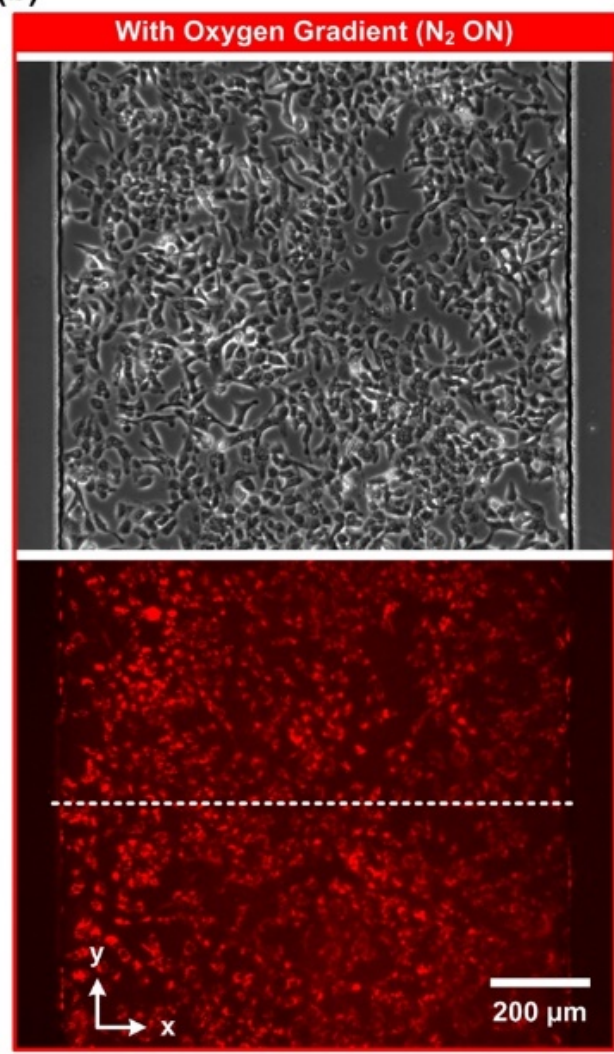

(c)

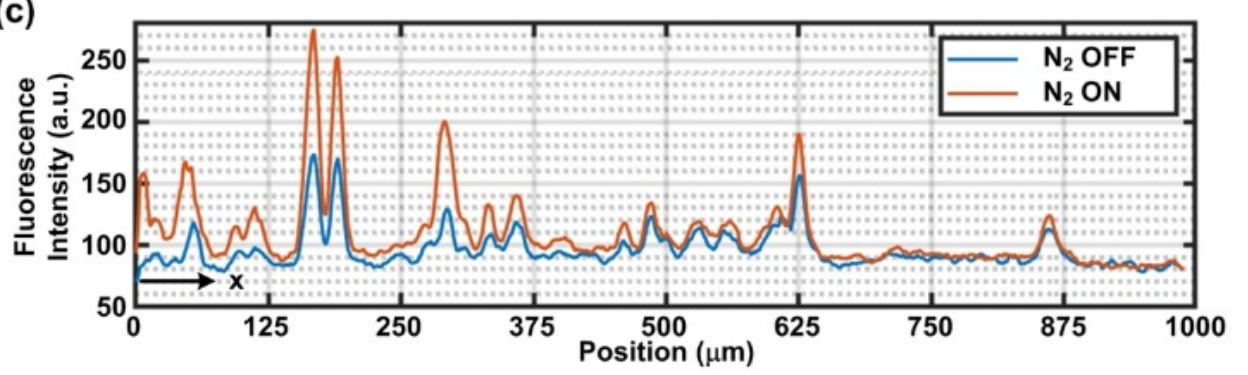

Figure 4. Phase constrast and fluorescence images of A549 cells treated with the intracellular oxygen sensitive reagent (MitoXpress Intra) under (a) normoxia $\left(\mathrm{N}_{2} \mathrm{OFF}\right)$ and (b) oxygen gradient $\left(\mathrm{N}_{2} \mathrm{ON}\right)$ conditions. (c) Representative line profiles of the fluorescence intensity across the width of the channel under the normoxia and oxygen gradient conditions. Comparing to the normoxia condition $\left(\mathrm{N}_{2}\right.$ $\mathrm{OFF})$, higher fluorescence intensity on the left-hand side under the oxygen gradient condition $\left(\mathrm{N}_{2} \mathrm{ON}\right)$ indicates lower intracellular oxygen tension within the A549 cells. 
(a)

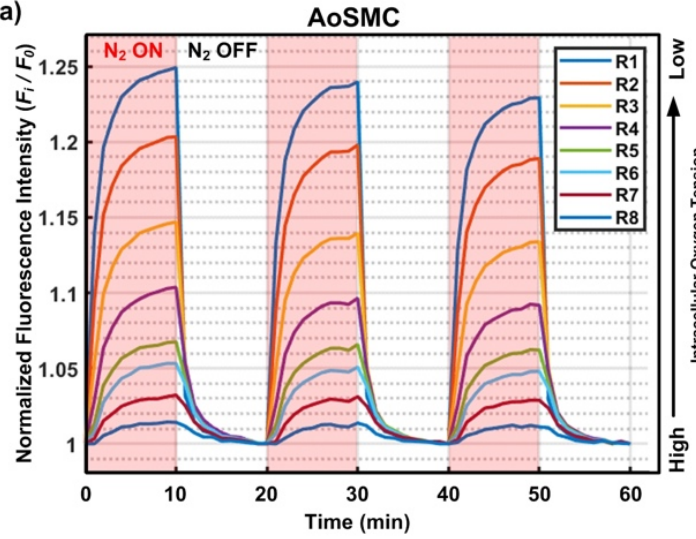

(c)

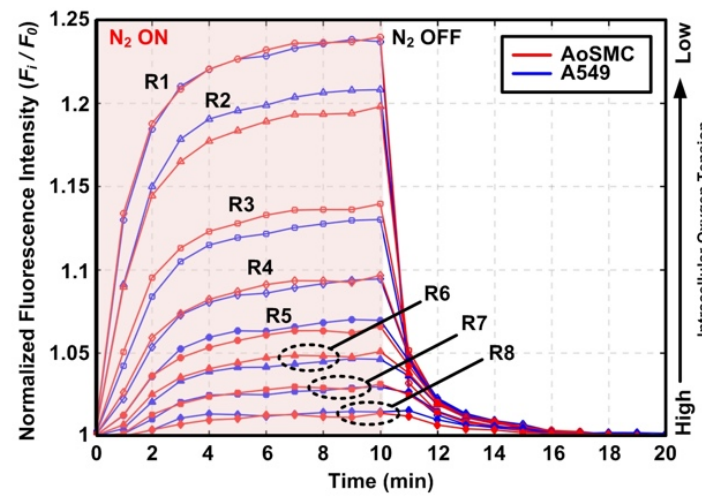

(e)

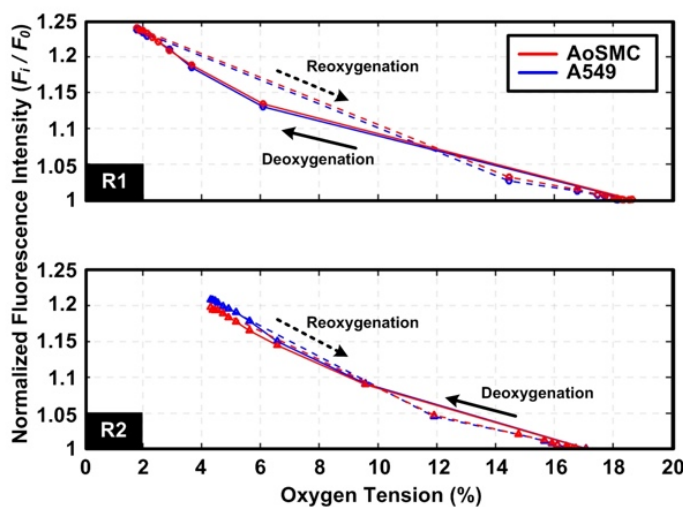

(b)

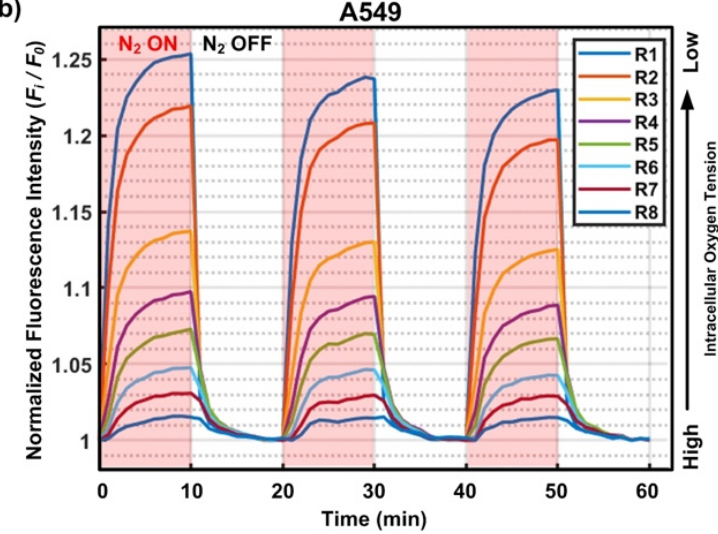

(d)
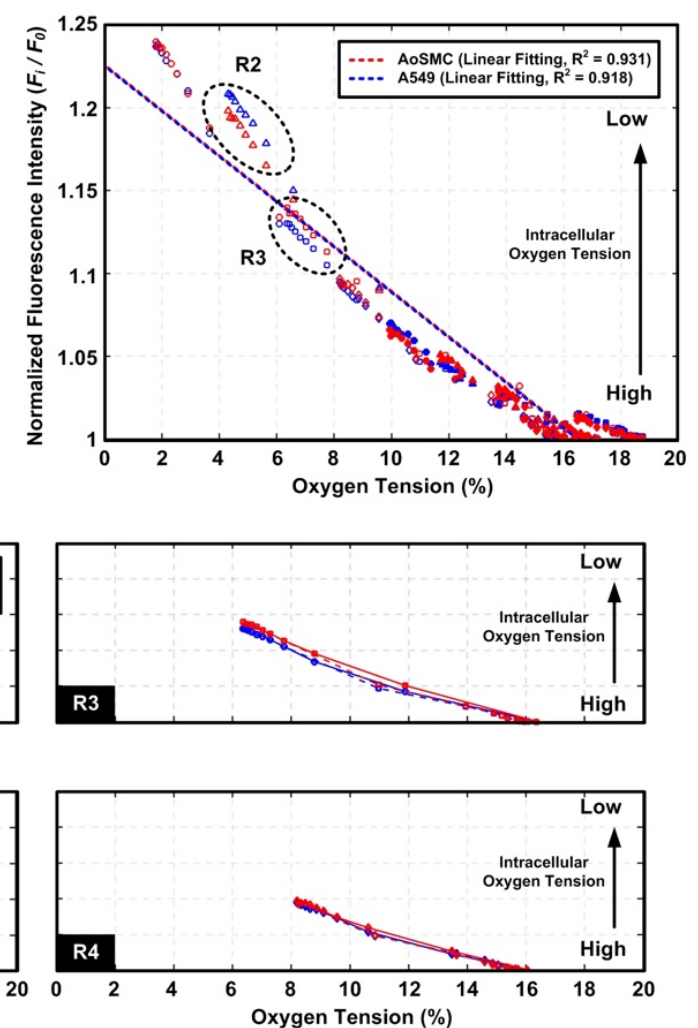

Figure 5. Temporal variation of the average normalized fluorescence intensities of the intracellular oxygen probes within the (a) AoSMC and (b) A549 cells located in different regions across the width of the cell culture channel (left to right is R1 to R8), respectively. Red background regions indicate the periods when nitrogen gas is on $\left(\mathrm{N}_{2} \mathrm{ON}\right)$ for the oxygen gradient generation. (c) Comparison of the average normalized fluorescence intensities of the intracellular oxygen probes within the AoSMC and A549 cells located in different regions ( $\mathrm{R} 1$ to $\mathrm{R} 8)$ during the period of one oxygen gradient/normoxia $\left(\mathrm{N}_{2}\right.$ $\mathrm{ON} / \mathrm{N}_{2} \mathrm{OFF}$ ) cycle. Intracellular oxygen probe shows discrepant fluorescence intensity between the AoSMC and A549 cells located in R2 and R3. (d) Plot of the normalized fluorescence intensities of the intracellular oxygen probes under different oxygen tensions generated in the microfluidic device for the cultured AoSMC and A549 cells. (e) The kinetics of the intracellular oxygen tension of the cells located in regions $\mathrm{R} 1$ to $\mathrm{R} 4$ during the deoxygenation and reoxygenation processes within a single period based on the fluorescence intensity observation. The solid and dotted lines show the intracellular oxygen tension responses during deoxygenation $\left(\mathrm{N}_{2} \mathrm{ON}\right)$ and reoxygenation $\left(\mathrm{N}_{2} \mathrm{OFF}\right)$ processes, respectively. 
(a)

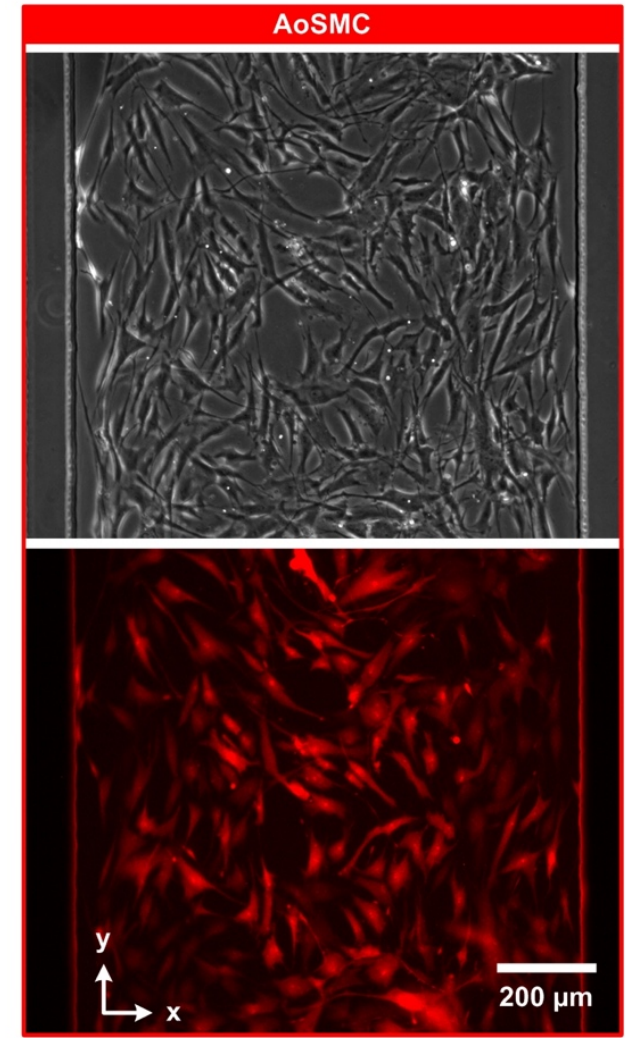

(b)

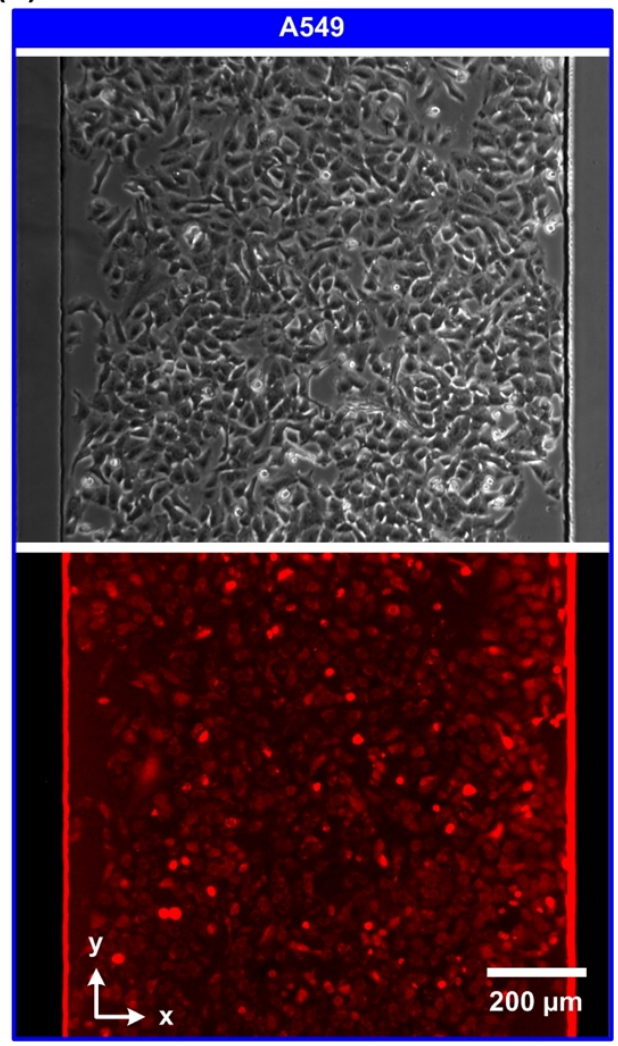

Figure 6. Phase contrast and fluorescence images of (a) AoSMC and (b) A549 cells cultured in the microfluidic devices and stained with X-Rhod-1 dye for intracellular calcium imaging. 

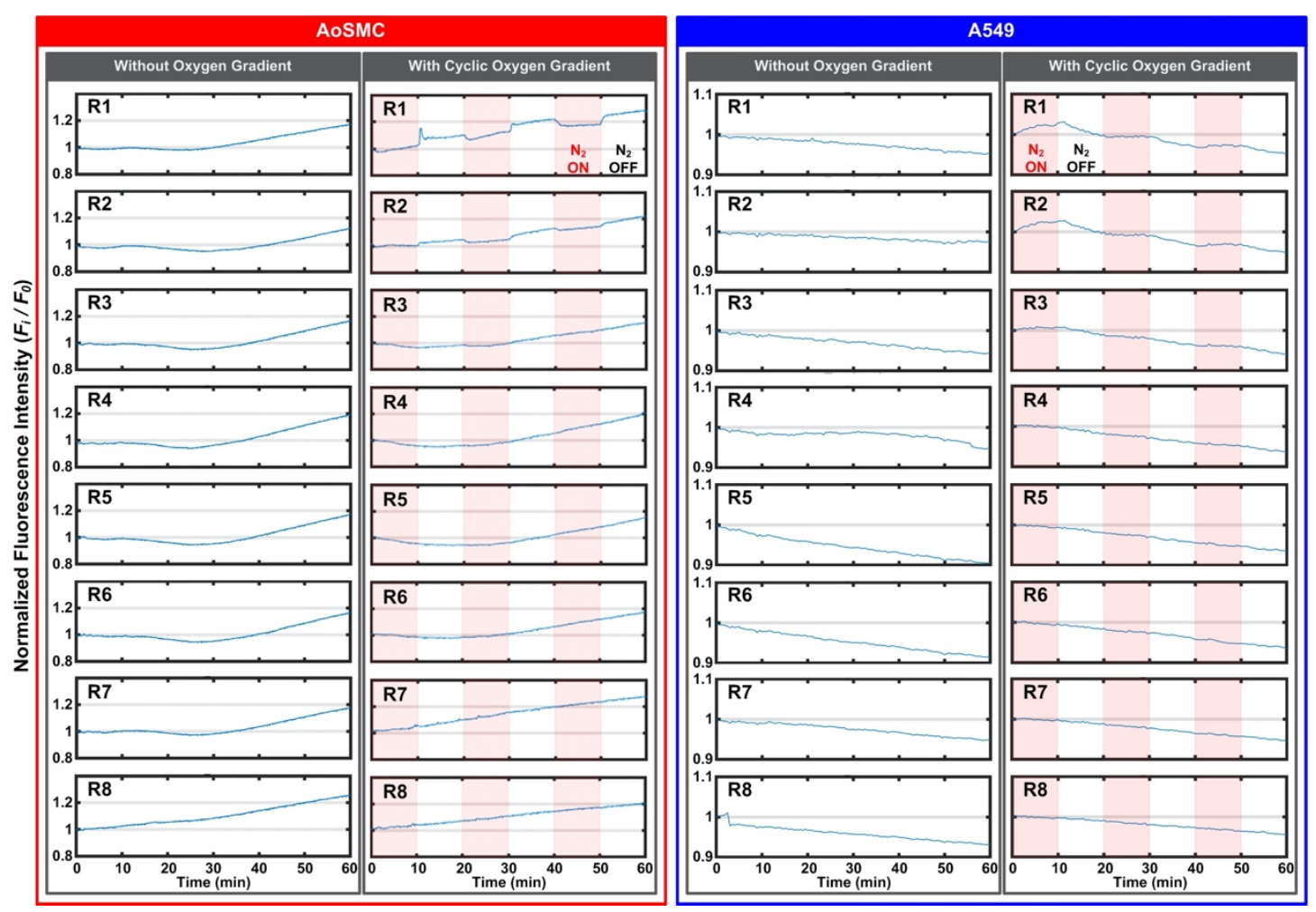

Figure 7. Temporal variation of intracellular calcium within the AoSMC and A549 cells located in different regions ( $\mathrm{R} 1$ to $\mathrm{R} 8$ ) based on the fluorescence intensity observation under normoxia $\left(\mathrm{N}_{2} \mathrm{OFF}\right)$ and oxygen gradient conditions $\left(\mathrm{N}_{2} \mathrm{ON}\right)$. Red background regions indicate the periods when nitrogen gas is on $\left(\mathrm{N}_{2} \mathrm{ON}\right)$ for the oxygen gradient generation. 
(a) Device Design

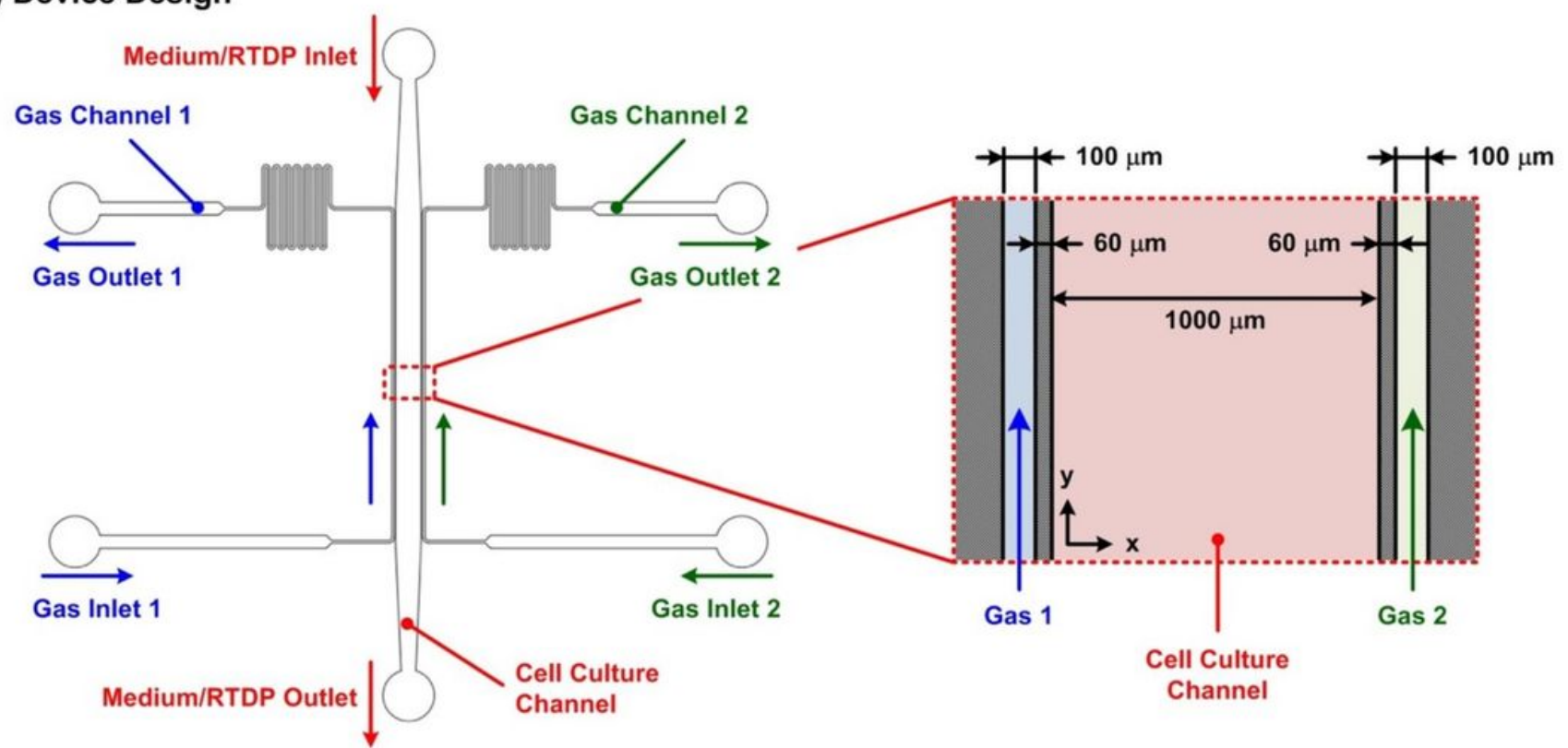

(b) Fabricated Device

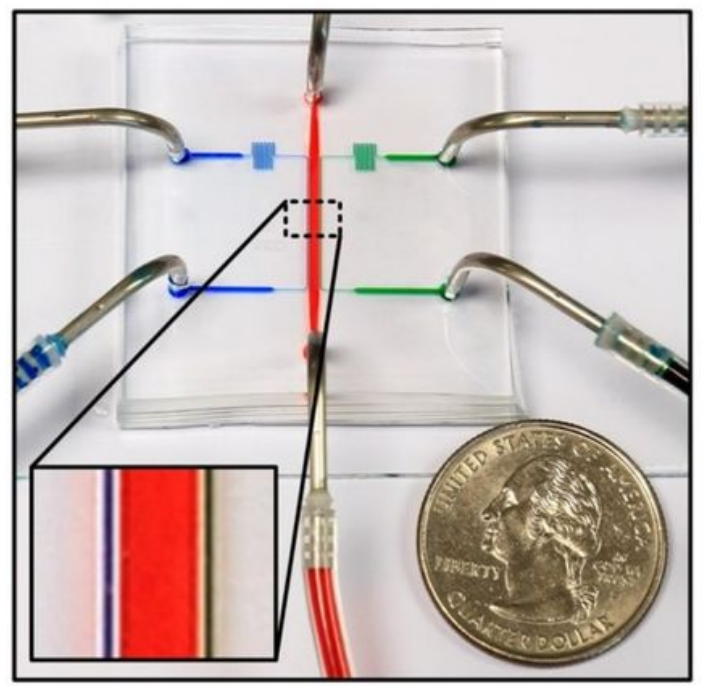

(c) Experimental Setup

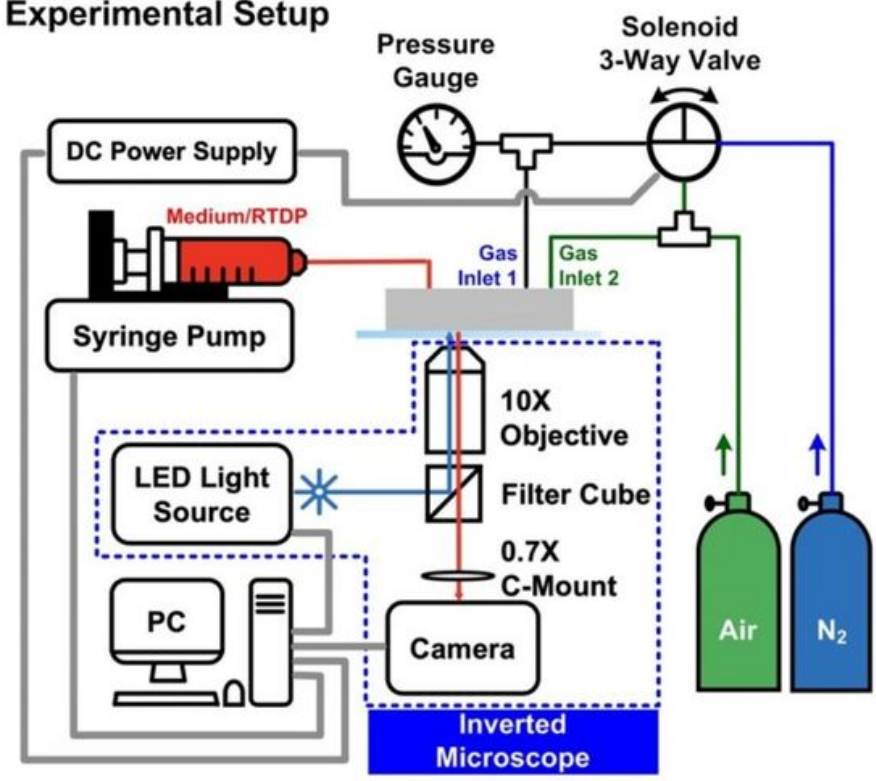

Figure 1

(a) Schematic of the PDMS microfluidic device capable of generating cyclic oxygen gradients. Three sets of microfluidic channels are designed in the device, including the middle cell culture channel and two side channels for oxygen gradient generation. The channels are separated by $60 \mu \mathrm{m}$-wide walls. Oxygen gradient in the cell culture channel is generated by purging pure nitrogen gas and air into the left (Inlet 1) and right (Inlet 2) side channels, respectively. (b) Experimental photos of the fabricated device filled with food dyes. (c) Experiment setup for generation of the cyclic oxygen gradients using the microfluidic device for cell culture experiments and observation. 
(a)

$\mathrm{N}_{2}$ OFF (Gas 1: Air; Gas 2: Air)

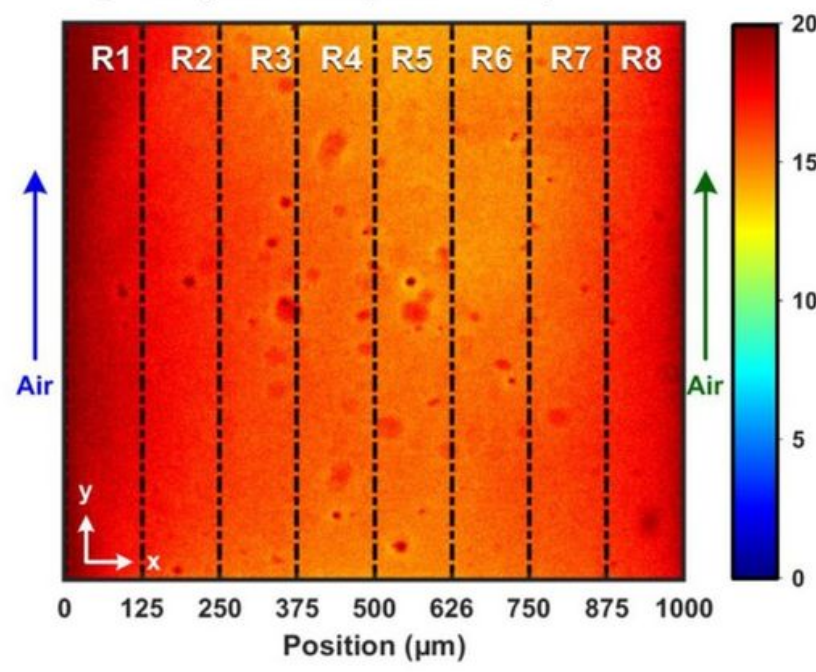

(c)

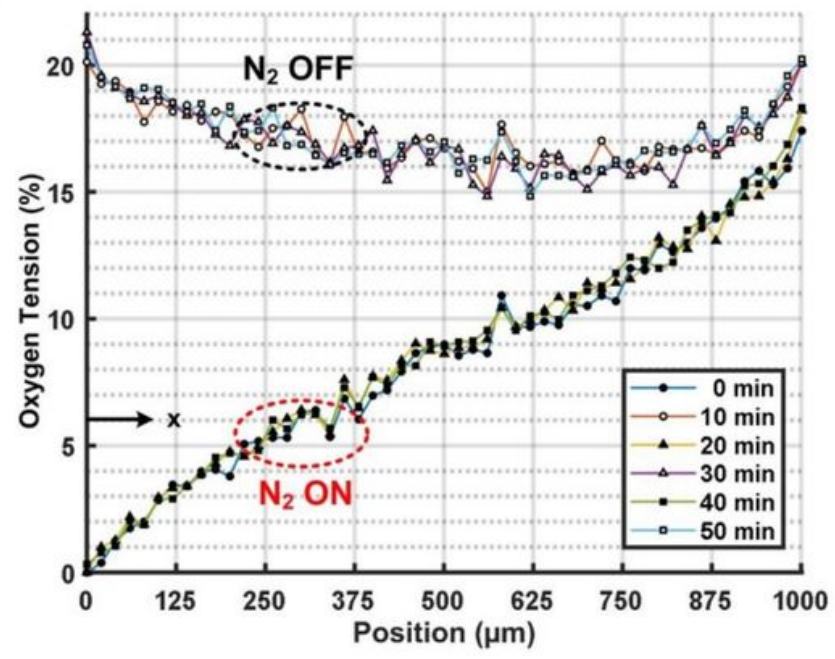

(b)

$\mathrm{N}_{2}$ ON (Gas 1: $\mathrm{N}_{2}$; Gas 2: Air)

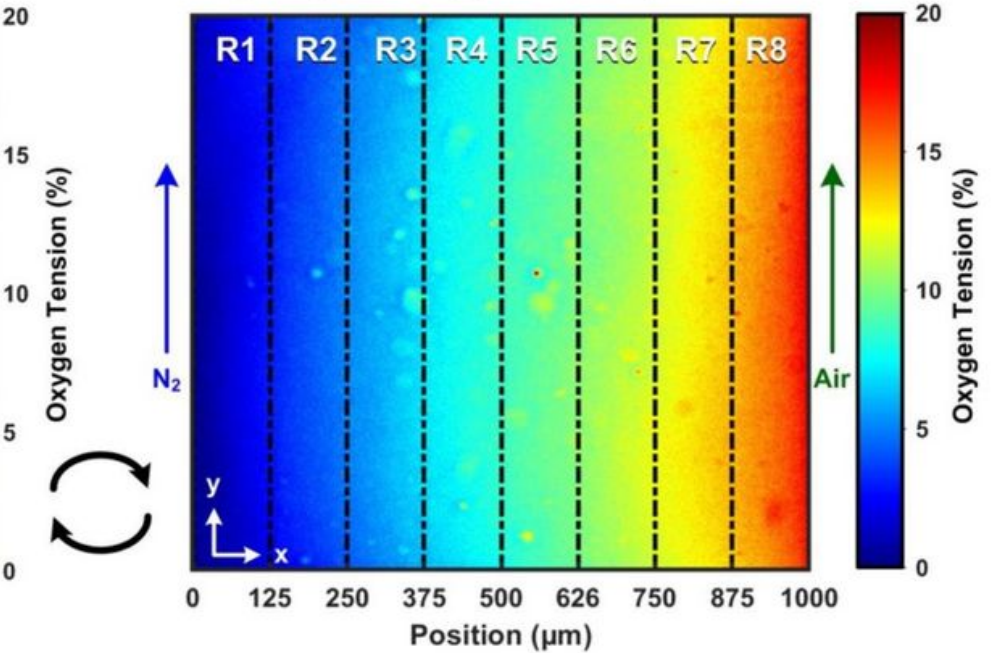

(d)

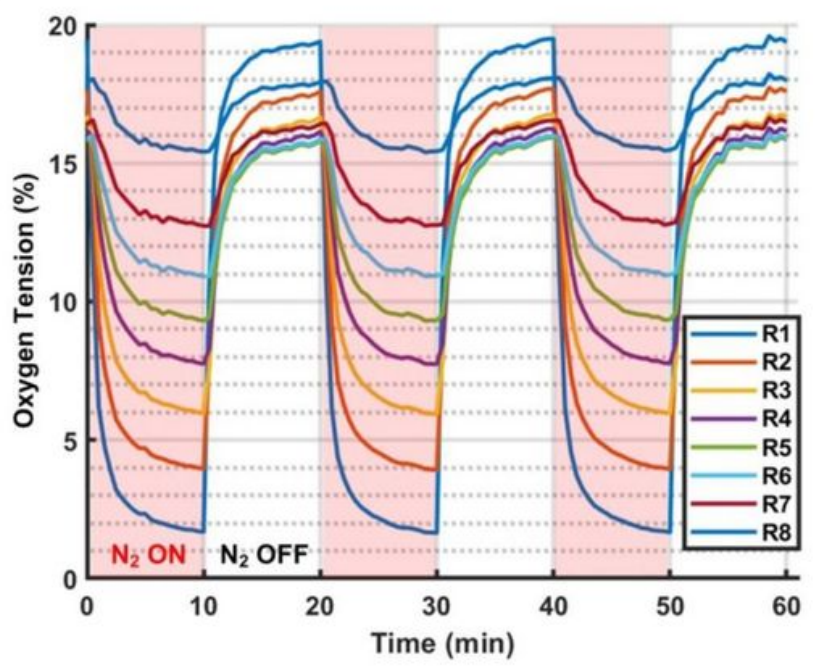

Figure 2

(a) and (b) are the characterized oxygen profiles without (N2 OFF) and with (N2 ON) oxygen gradients, respectively. (c) Line profiles show the consistent oxygen gradients (solid symbol lines) can be established when nitrogen gas is on ( $\mathrm{N} 2 \mathrm{ON}$ ), and relative uniform normoxia conditions (hollow symbol lines) can be recovered by air purging (N2 OFF). (d) The temporal variation of average oxygen tension values from the leftmost (R1) to the rightmost region (R8). Cyclic oxygen tension profiles in different regions show that the oxygen tensions are oscillated in the period of 20 minutes (10 min on/10 min off). 
(a)

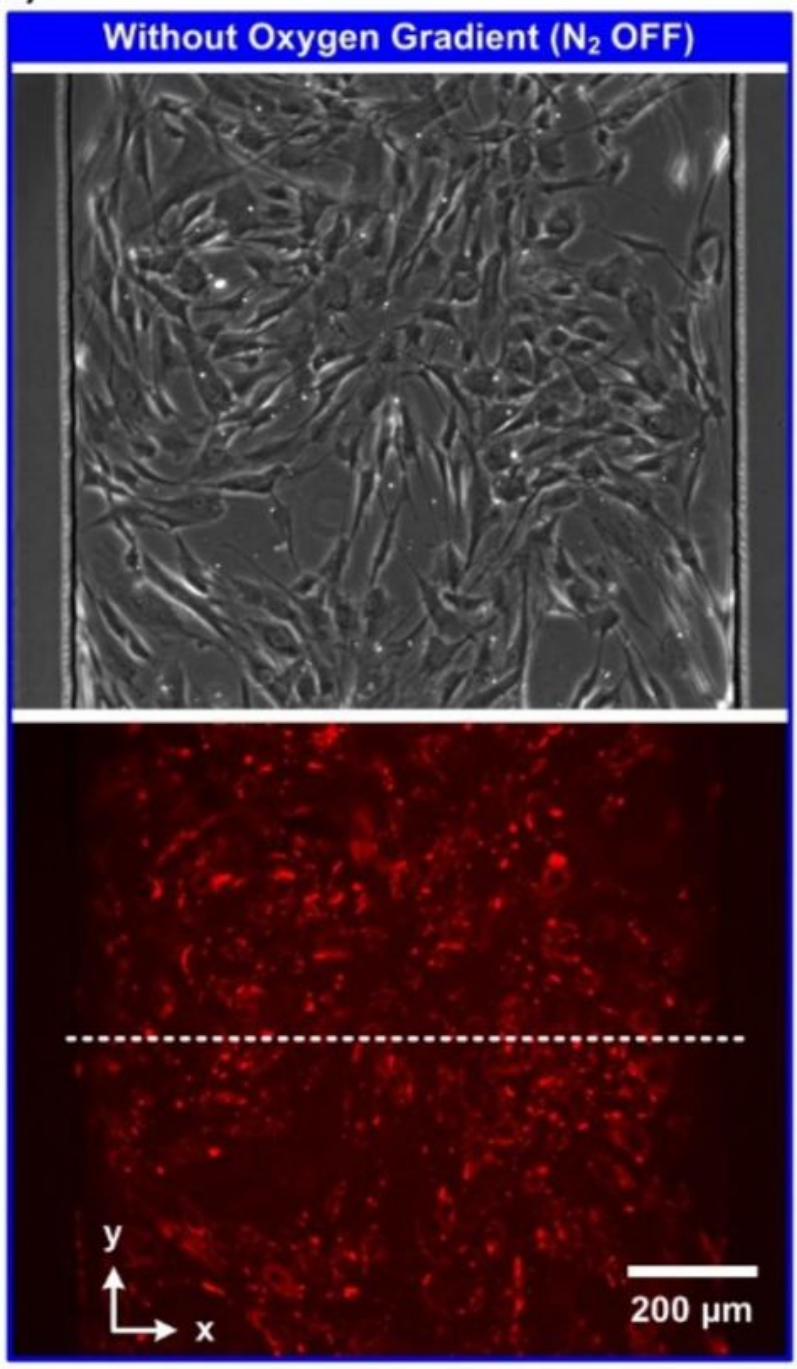

(b)

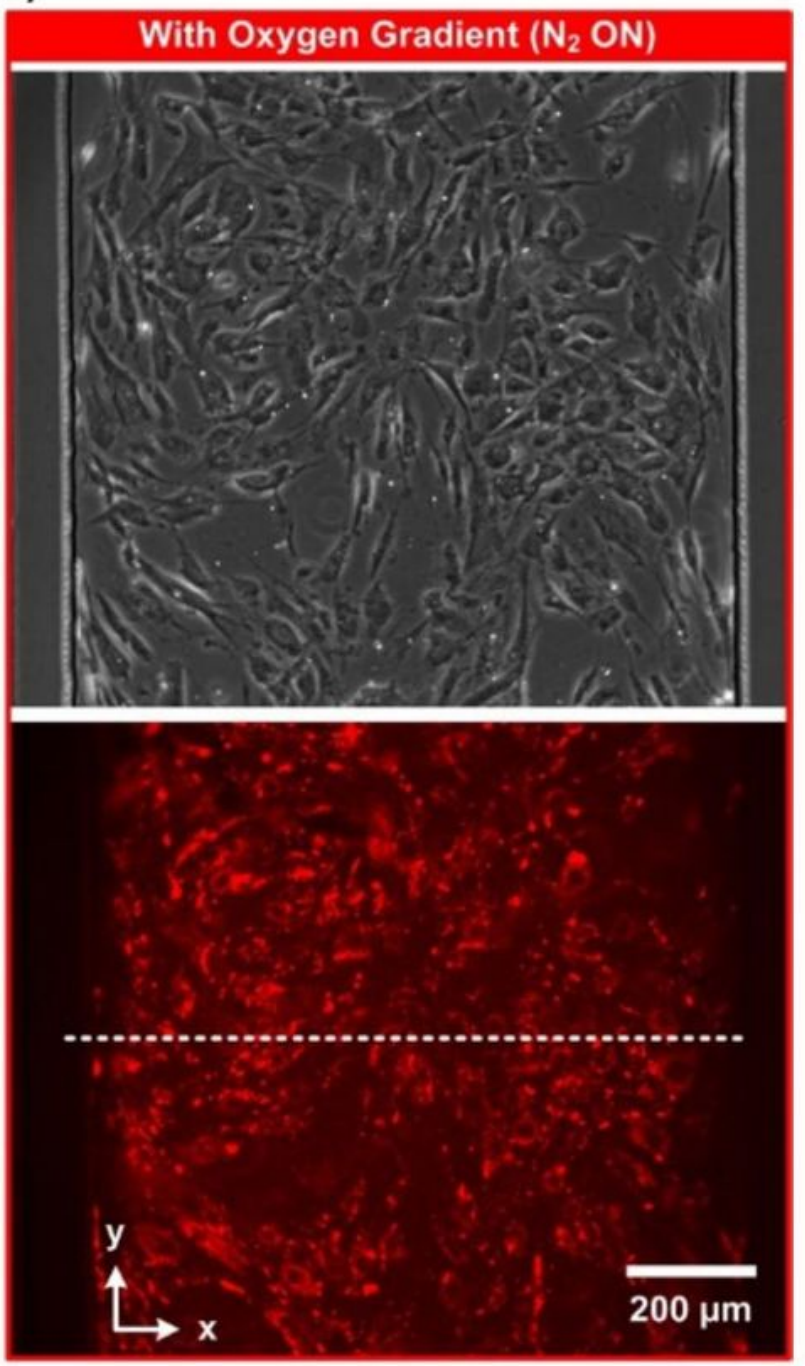

(c)

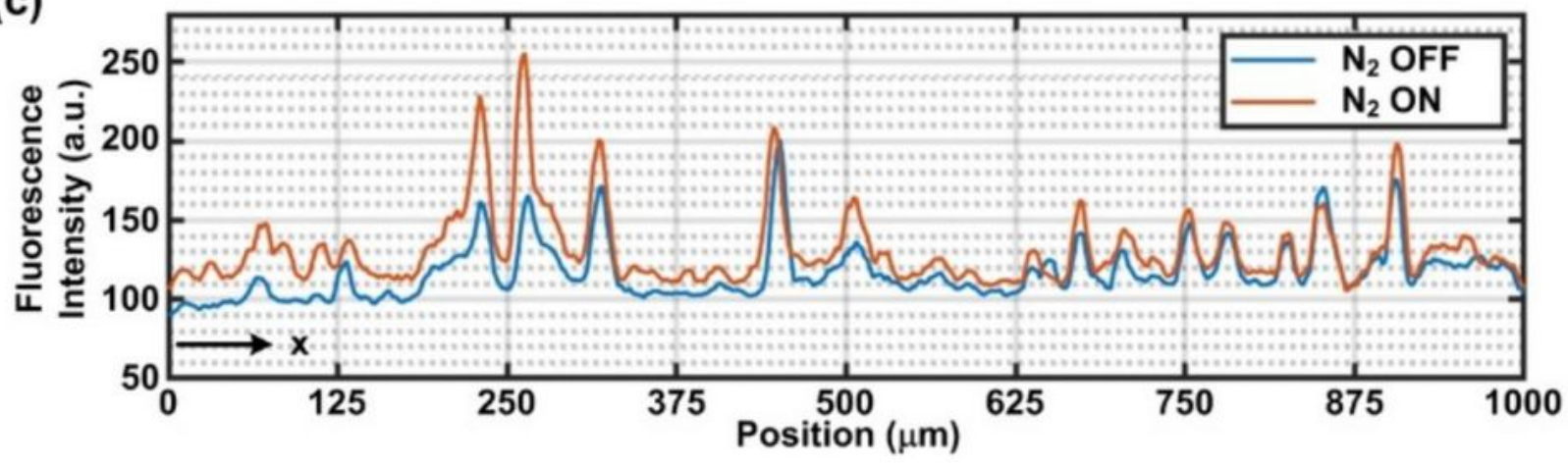

Figure 3

Bright field and fluorescence images of AoSMC treated with the intracellular oxygen sensitive reagent (MitoXpress Intra) under (a) normoxia (N2 OFF) and (b) oxygen gradient (N2 ON) conditions. (c) Representative line profiles of the fluorescence intensity across the width of the channel under the normoxia and oxygen gradient conditions. Comparing to the normoxia condition (N2 OFF), higher 
fluorescence intensity on the left-hand side under the oxygen gradient condition (N2 ON) indicates lower intracellular oxygen tension within the AoSMCs.

(a)

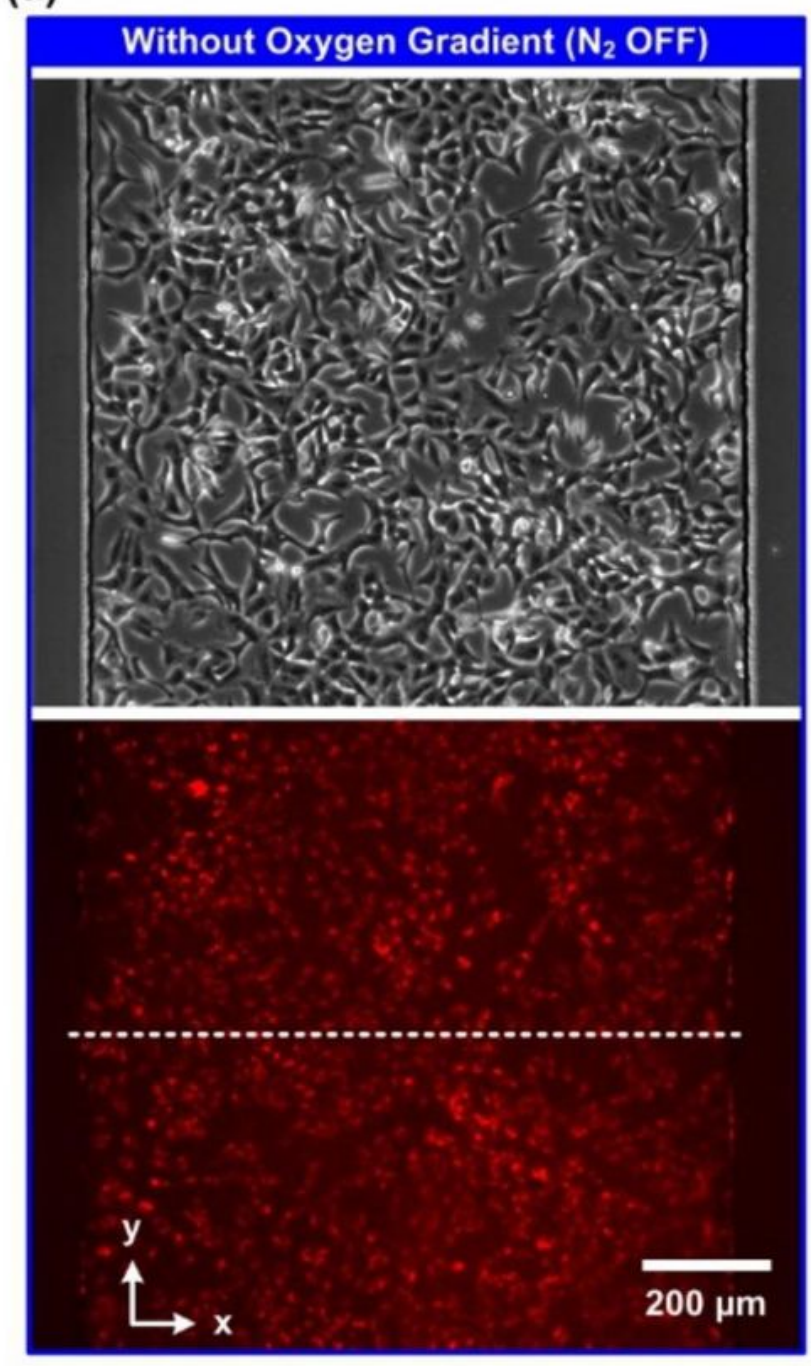

(b)

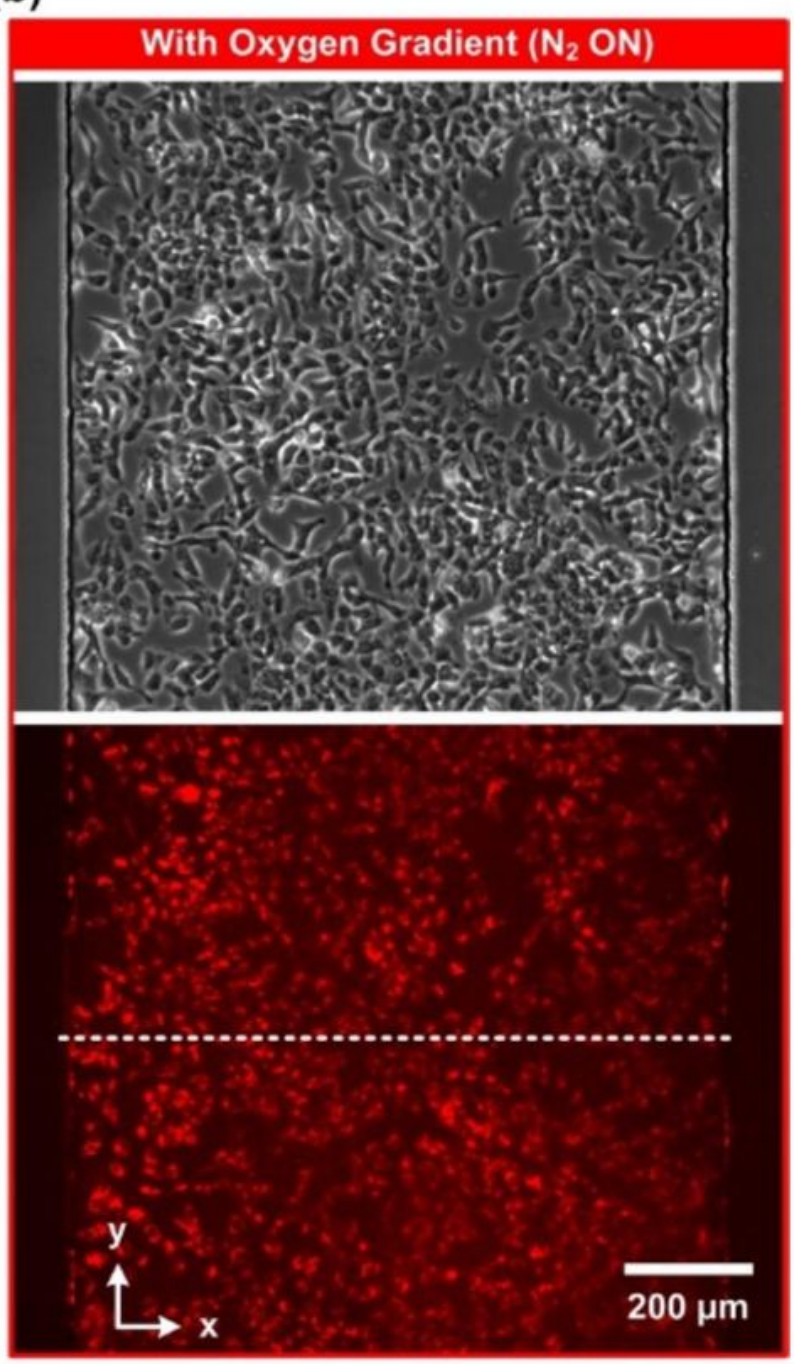

(c)

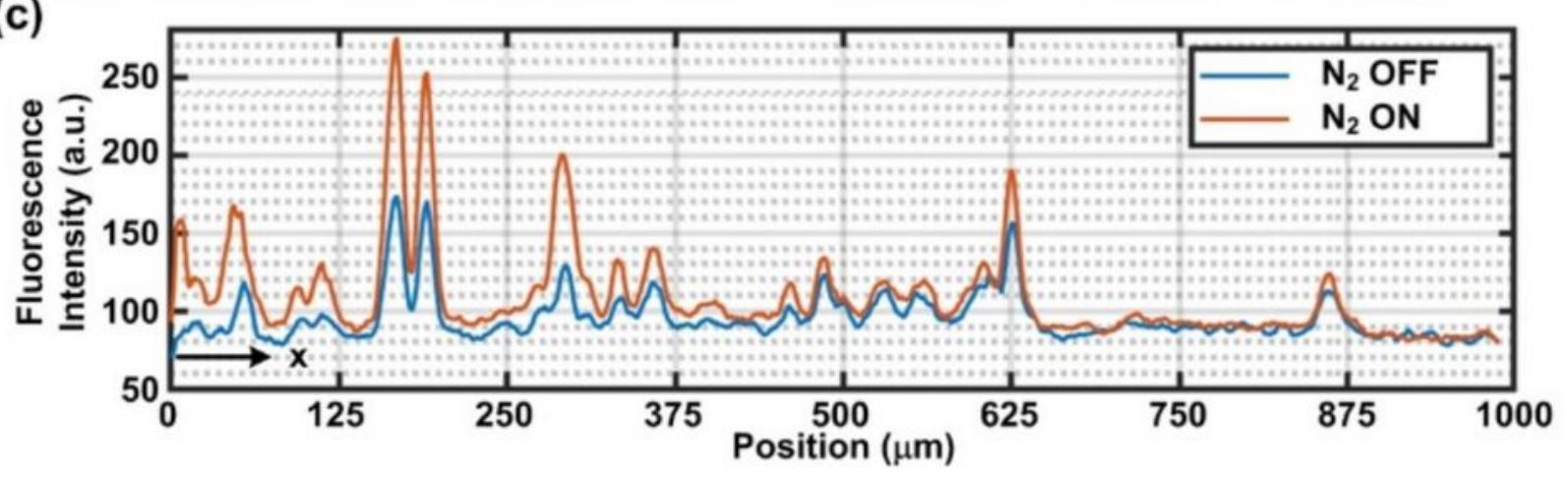

\section{Figure 4}

Phase constrast and fluorescence images of A549 cells treated with the intracellular oxygen sensitive reagent (MitoXpress Intra) under (a) normoxia (N2 OFF) and (b) oxygen gradient (N2 ON) conditions. (c) Representative line profiles of the fluorescence intensity across the width of the channel under the 
normoxia and oxygen gradient conditions. Comparing to the normoxia condition (N2 OFF), higher fluorescence intensity on the left-hand side under the oxygen gradient condition (N2 ON) indicates lower intracellular oxygen tension within the A549 cells.

(a)

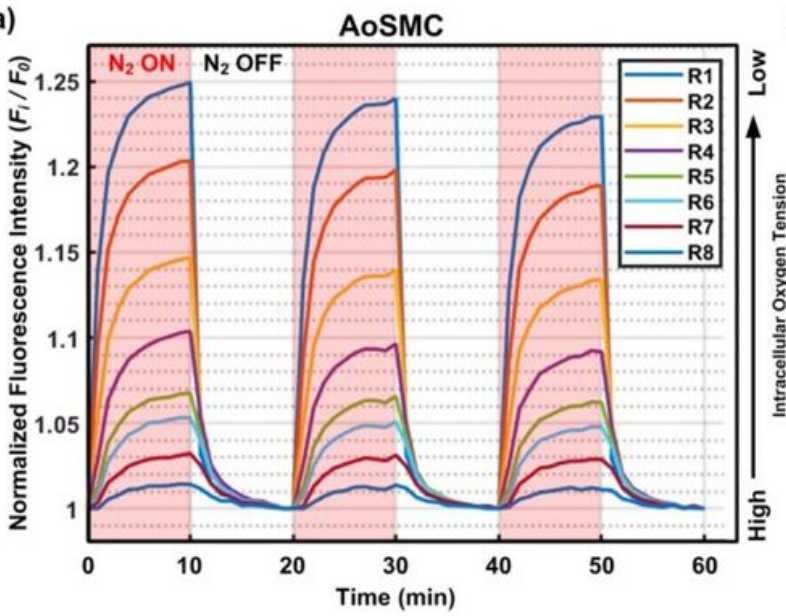

(c)

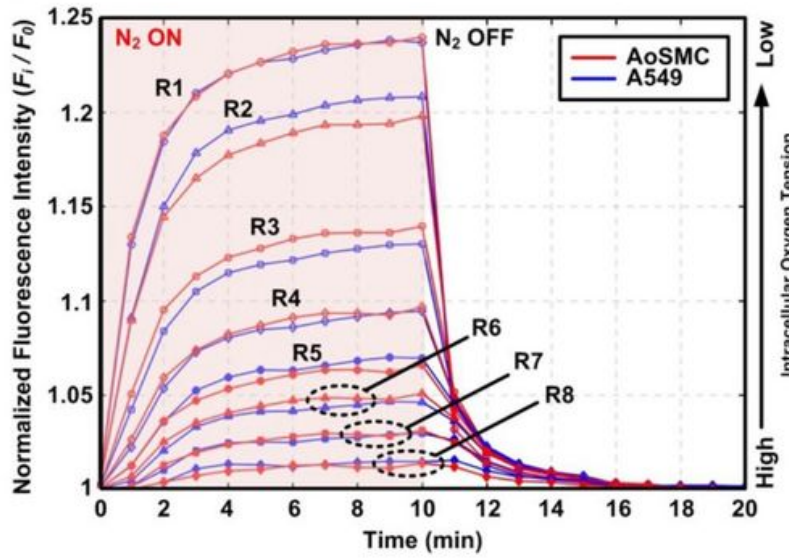

(e)

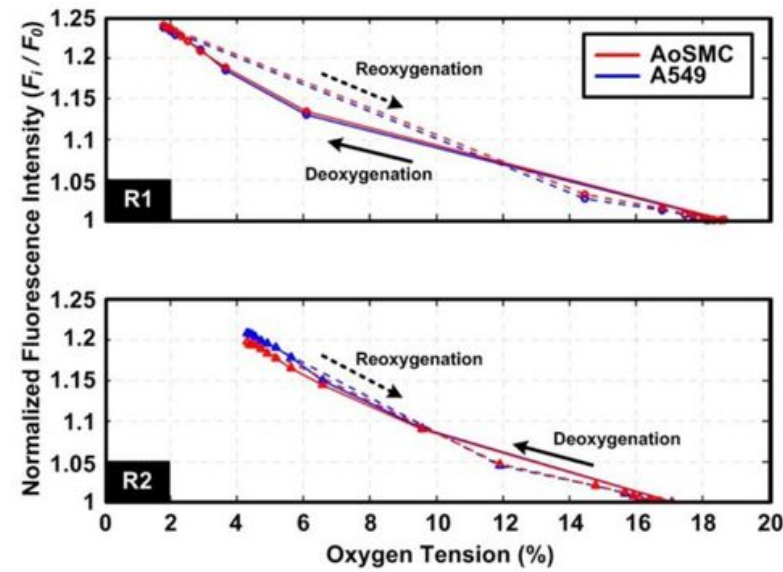

(b)

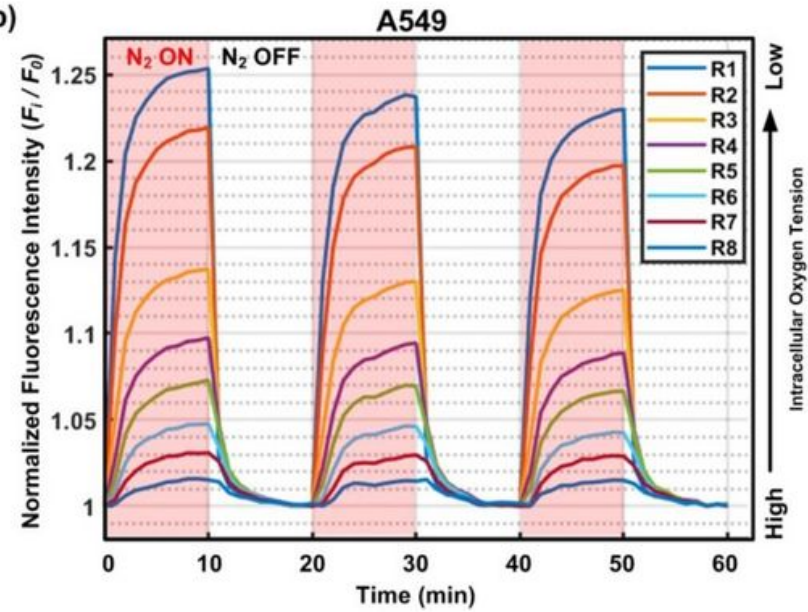

(d)
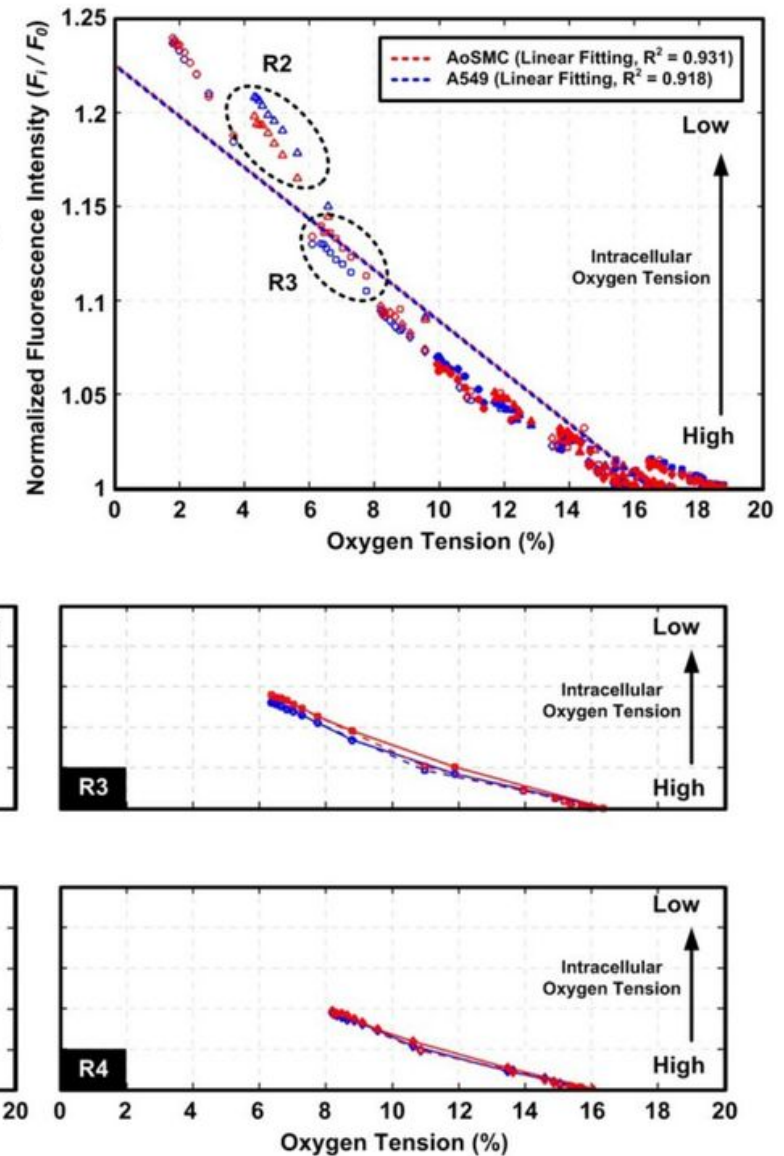

\section{Figure 5}

Temporal variation of the average normalized fluorescence intensities of the intracellular oxygen probes within the (a) AoSMC and (b) A549 cells located in different regions across the width of the cell culture channel (left to right is R1 to R8), respectively. Red background regions indicate the periods when nitrogen gas is on (N2 ON) for the oxygen gradient generation. (c) Comparison of the average normalized 
fluorescence intensities of the intracellular oxygen probes within the AoSMC and A549 cells located in different regions (R1 to R8) during the period of one oxygen gradient/normoxia (N2 ON/N2 OFF) cycle. Intracellular oxygen probe shows discrepant fluorescence intensity between the AoSMC and A549 cells located in R2 and R3. (d) Plot of the normalized fluorescence intensities of the intracellular oxygen probes under different oxygen tensions generated in the microfluidic device for the cultured AoSMC and A549 cells. (e) The kinetics of the intracellular oxygen tension of the cells located in regions R1 to R4 during the deoxygenation and reoxygenation processes within a single period based on the fluorescence intensity observation. The solid and dotted lines show the intracellular oxygen tension responses during deoxygenation (N2 ON) and reoxygenation (N2 OFF) processes, respectively.

(a)
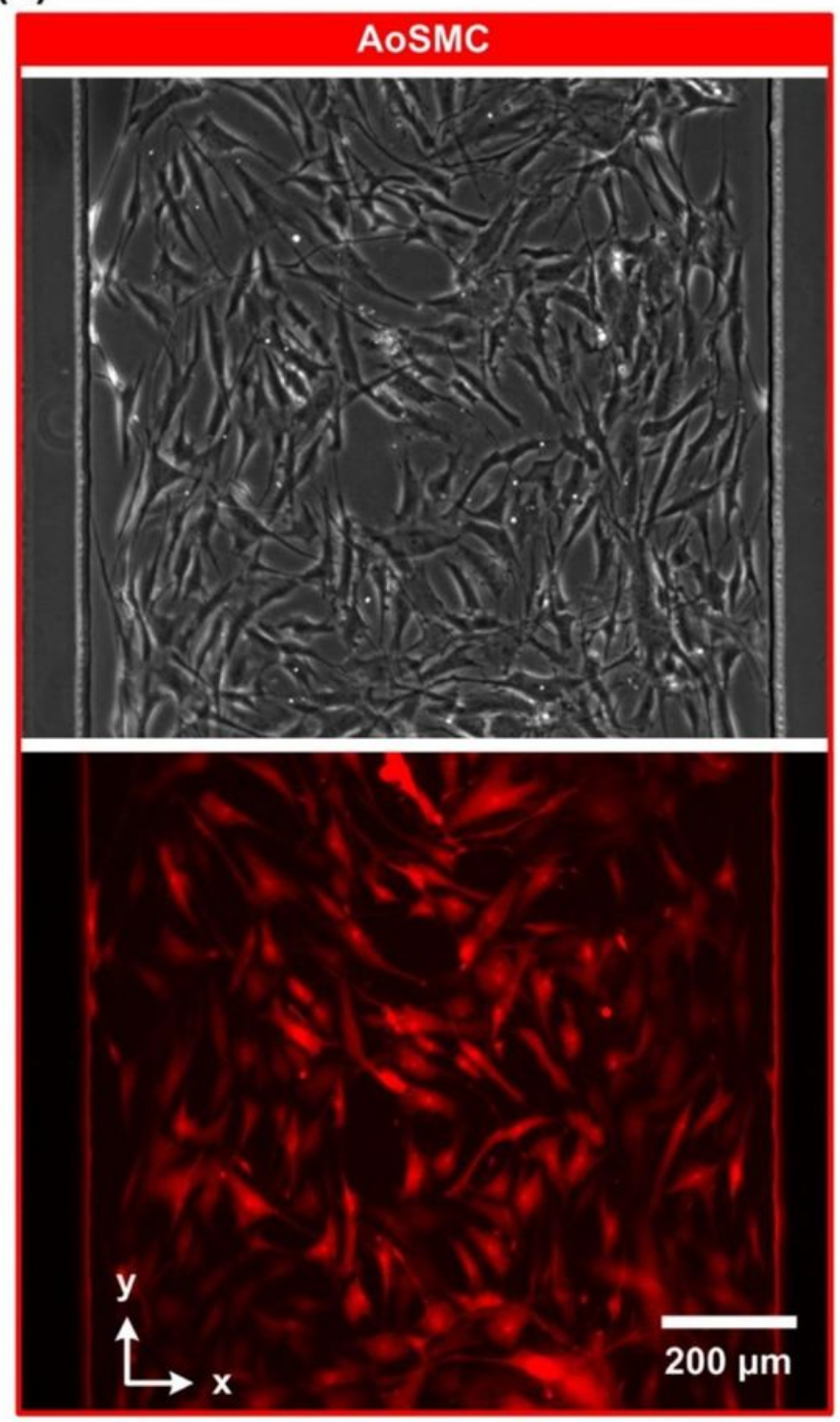

(b)

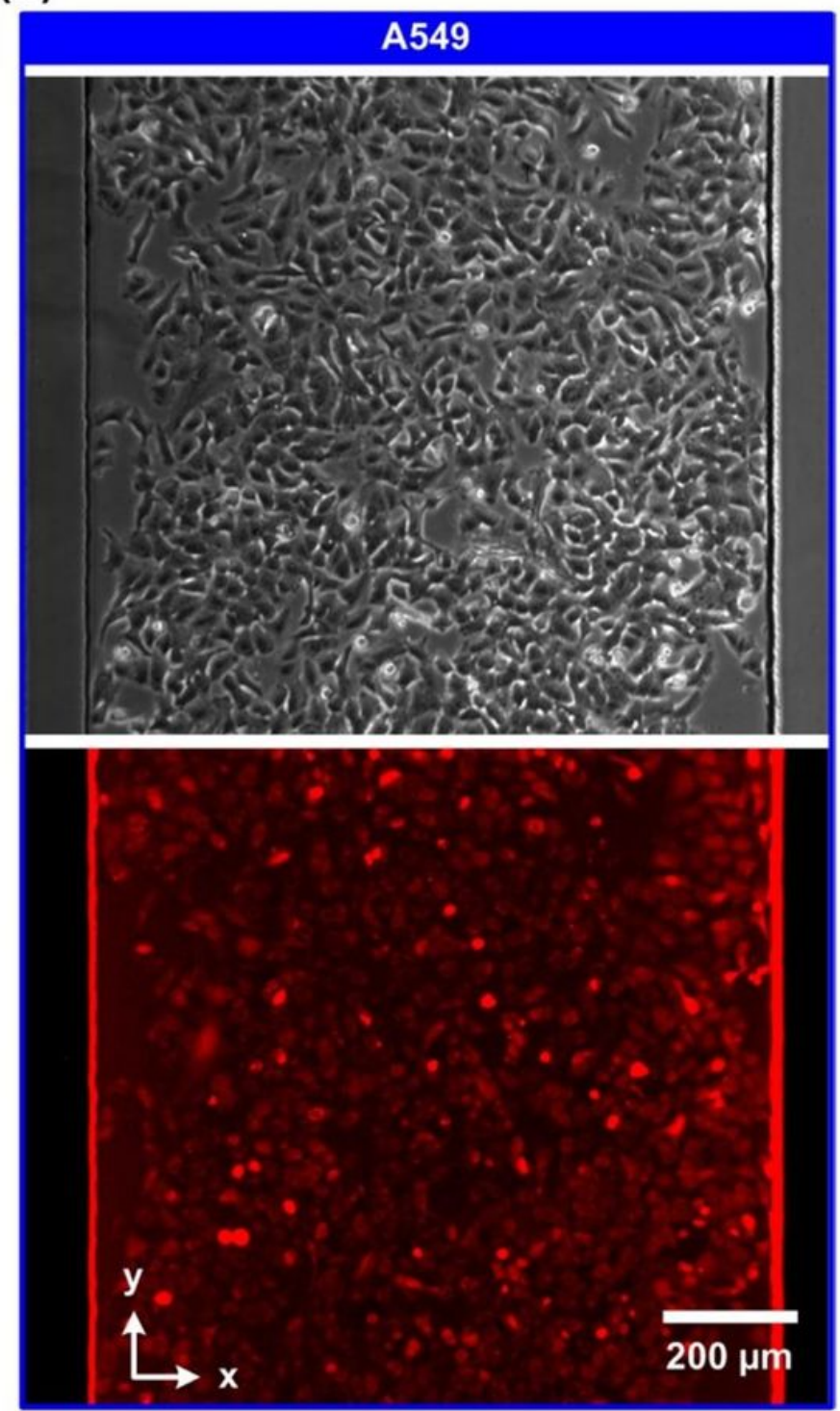

\section{Figure 6}

Phase contrast and fluorescence images of (a) AoSMC and (b) A549 cells cultured in the microfluidic devices and stained with X-Rhod-1 dye for intracellular calcium imaging. 

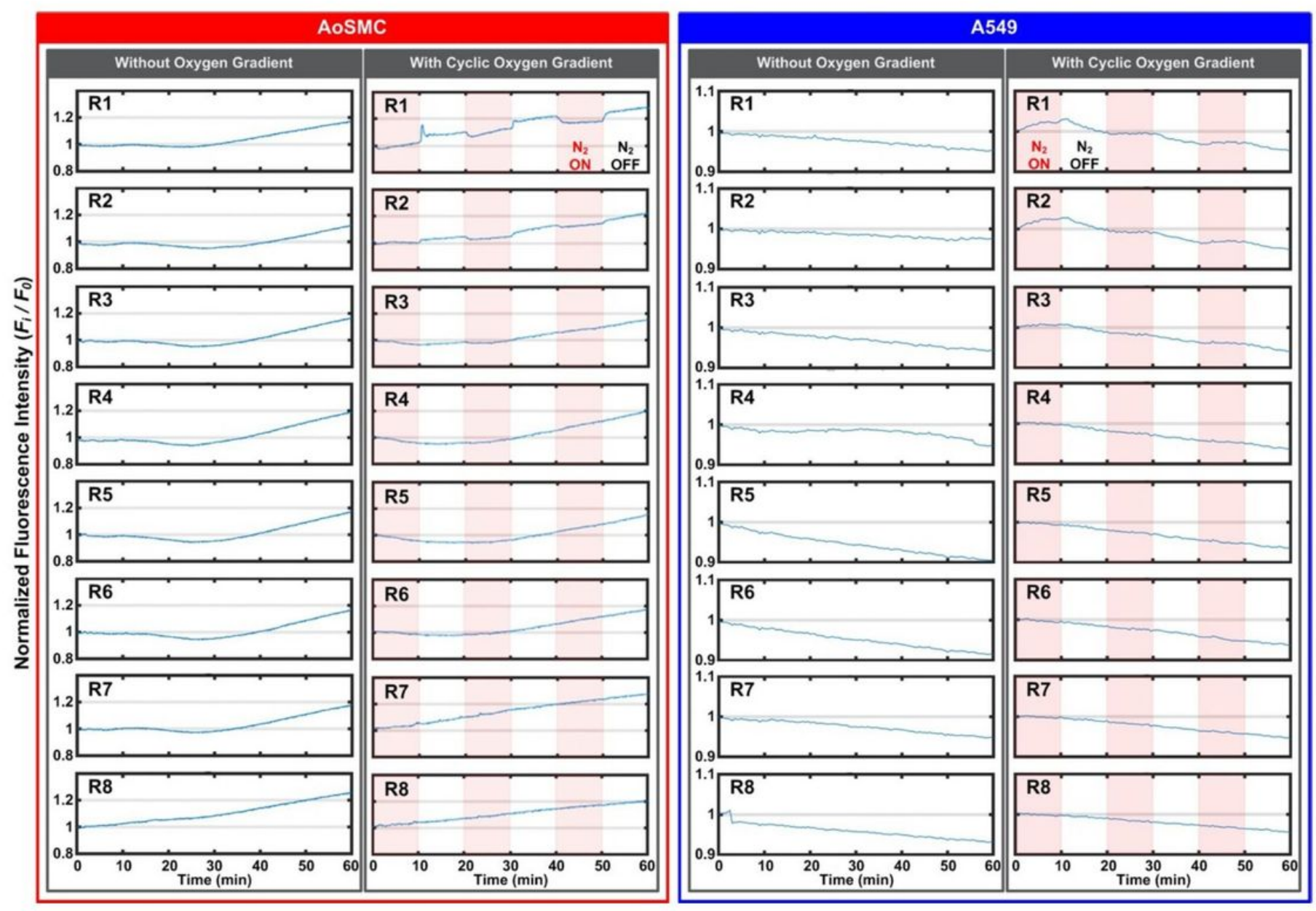

\section{Figure 7}

Temporal variation of intracellular calcium within the AoSMC and A549 cells located in different regions (R1 to R8) based on the fluorescence intensity observation under normoxia (N2 OFF) and oxygen gradient conditions ( $22 \mathrm{ON}$ ). Red background regions indicate the periods when nitrogen gas is on (N2 ON) for the oxygen gradient generation

\section{Supplementary Files}

This is a list of supplementary files associated with this preprint. Click to download.

- oxygentension.avi 